\title{
Equivalence of Non-Perfect Secret Sharing and Symmetric Private Information Retrieval with General Access Structure
}

\author{
Seunghoan Song, Member, IEEE and Masahito Hayashi, Fellow, IEEE
}

\begin{abstract}
We study the equivalence between non-perfect secret sharing (NSS) and symmetric private information retrieval (SPIR) with arbitrary response and collusion patterns. NSS and SPIR are defined with an access structure, which corresponds to the authorized/forbidden sets for NSS and the response/collusion patterns for SPIR. We prove the equivalence between NSS and SPIR in the following two senses. 1) Given any SPIR protocol with an access structure, an NSS protocol is constructed with the same access structure and the same rate. 2) Given any linear NSS protocol with an access structure, a linear SPIR protocol is constructed with the same access structure and the same rate. We prove the first relation even if the SPIR protocol has imperfect correctness and secrecy. From the first relation, we derive an upper bound of the SPIR capacity for arbitrary response and collusion patterns. For the special case of $n$-server SPIR with $r$ responsive and $t$ colluding servers, this upper bound proves that the SPIR capacity is $(r-t) / n$. From the second relation, we prove that a SPIR protocol exists for any response and collusion patterns.
\end{abstract}

\section{INTRODUCTION}

\section{A. Nonperfect Secret Sharing and Symmetric Private Informa- tion Retrieval}

Secret sharing (SS) and private information retrieval (PIR) are two extensively studied cryptographic protocols. SS [2], [3] considers the problem in which a dealer encodes a secret into $\mathrm{n}$ shares so that some subsets of shares can reconstruct the secret but the other subsets have no information of the secret. PIR [4] considers the problem in which a user retrieves one of the multiple files from server(s) without revealing which file is retrieved. Since PIR with one server has no efficient solution [4], it has been extensively studied with multiple noncommunicating servers, and thus, in the following, we simply denote multi-server PIR by PIR. SS and PIR have a similar

S. Song is supported by JSPS Grant-in-Aid for JSPS Fellows No. JP20J11484. M. Hayashi is supported in part by Guangdong Provincial Key Laboratory (Grant No. 2019B121203002), a JSPS Grant-in-Aids for Scientific Research (A) No.17H01280 and for Scientific Research (B) No.16KT0017, and Kayamori Foundation of Information Science Advancement. This article was presented in part at Proceedings of 2021 IEEE International Symposium on Information Theory [1].

S. Song is with Graduate school of Mathematics, Nagoya University, Nagoya, 464-8602, Japan (e-mail: m17021a@math.nagoya-u.ac.jp).

M. Hayashi is with Shenzhen Institute for Quantum Science and Engineering, Southern University of Science and Technology, Shenzhen, 518055, China, Guangdong Provincial Key Laboratory of Quantum Science and Engineering, Southern University of Science and Technology, Shenzhen 518055, China, Shenzhen Key Laboratory of Quantum Science and Engineering, Southern University of Science and Technology, Shenzhen 518055, China, and Graduate School of Mathematics, Nagoya University, Nagoya, 464-8602, Japan (e-mail:hayashi@sustech.edu.cn).

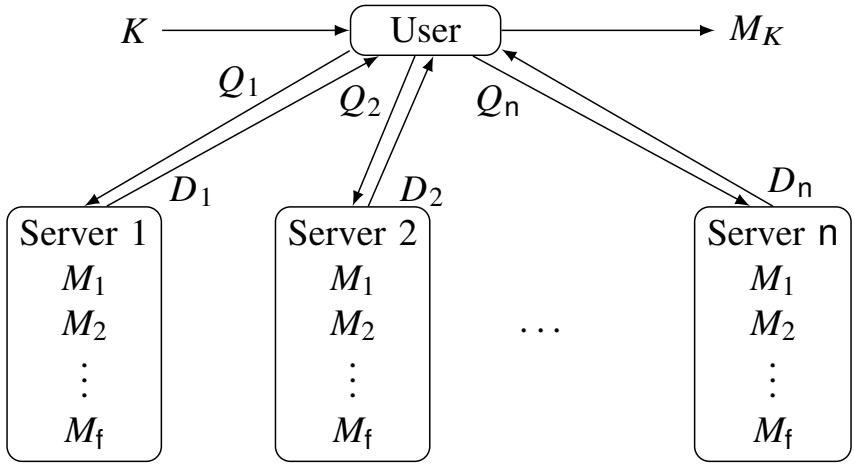

(a) SPIR Protocol

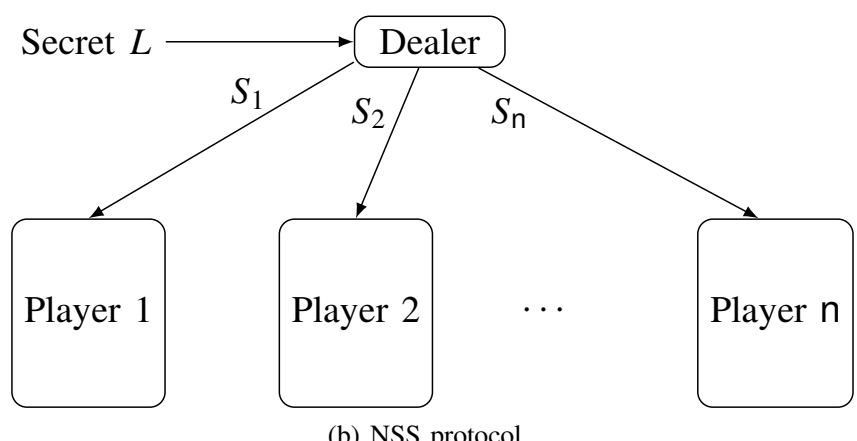

(b) NSS protocol

Fig. 1. NSS protocol and SPIR protocol with access structure $(\mathfrak{A}, \mathfrak{B})$. Let $\mathrm{n}=3, \mathfrak{A}=\{\{2,3\},\{1,2,3\}\}$, and $\mathfrak{B}=\{\emptyset,\{1\},\{2\},\{3\},\{1,2\}\}$. Since $\{2,3\} \in \mathfrak{A}, M_{K}(L)$ can be reconstructed from the answers $D_{2}, D_{3}$ (shares $\left.S_{2}, S_{3}\right)$. Since $\{1,2\} \in \mathfrak{B}$, no information of $K(L)$ can be extracted from the answers $D_{1}, D_{2}$ (shares $S_{1}, S_{2}$ ).

structure because the secrecy of both protocols is obtained by partitioning the confidential information. On the other hand, the two protocols have a different structure because in SS, the secret is both the confidential and targeted information but in PIR, the targeted file is not confidential. From the similarity, there have been several studies to construct PIR from secret sharing [5], [6], [7], [8], [9]. However, the relation between these two protocols has not been clearly discussed.

In this paper, we study the relation between an extended class of SS, called non-perfect SS (NSS), and that of PIR, called symmetric PIR (SPIR). NSS is first discussed by [10], [11] with thresholds and is extended for general access structures [12], [13], [14], [15], [16]. NSS with general access structures is defined with two collections $\mathfrak{A}, \mathfrak{B} \subset 2^{[\mathrm{n}]}:=$ $2^{\{1, \ldots, n\}}$ which represent the authorized sets and the forbidden sets, respectively. The shares indexed by any element of $\mathfrak{A}$ can 
TABLE I

COMPARISON OF SPIR AND NSS

\begin{tabular}{|c|c|c|}
\hline & SPIR & NSS \\
\hline Messages & f files & one secret \\
\hline Participants & one user \& $\mathrm{n}$ servers & one dealer \& $\mathrm{n}$ players \\
\hline \multirow{3}{*}{ Security constraints } & Correctness & Correctness \\
& Server secrecy & Secrecy \\
& User secrecy & \\
\hline Communication & Two-way & One-way \\
\hline
\end{tabular}

reconstruct the secret but those indexed by any element of $\mathfrak{B}$ have no information of the secret. We denote NSS with $\mathfrak{A}$ and $\mathfrak{B}$ by $(\mathfrak{A}, \mathfrak{B}, \mathrm{n})$-NSS. The (perfect) SS corresponds to the $(\mathfrak{A}, \mathfrak{B}, \mathrm{n})$-NSS with $\mathfrak{A} \cup \mathfrak{B}=2^{[\mathrm{n}]}$.

SPIR [5] is a variant of PIR in which the user only obtains the targeted file but no information of the other files. The paper [5] proved that shared randomness of servers is necessary to achieve SPIR. This paper considers SPIR with arbitrary response and collusion patterns $\mathfrak{A}, \mathfrak{B} \subset 2^{[n]}$. We define a $(\mathfrak{A}, \mathfrak{B}, n, f)$-SPIR protocol by an $n$-server $f$-file protocol with the following conditions: i) the user correctly recovers the targeted file even if only the servers indexed by $\mathcal{A} \in \mathfrak{A}$ answer to the user; ii) the identity of the retrieved file is not leaked even if the servers indexed by $\mathcal{B} \in \mathfrak{B}$ collude; and iii) the user obtains no other information than the targeted file. If $\mathfrak{A}=\{[\mathrm{n}]\}$ and $\mathfrak{B}=\{\{i\} \in i \in[\mathrm{n}]\},(\mathfrak{H}, \mathfrak{B}, \mathrm{n}, \mathrm{f})$-SPIR is the SPIR in which no servers collude and all servers respond, which has been discussed in [5], [17].

The efficiency of a PIR protocol is evaluated by the PIR rate

$$
R_{\mathrm{PIR}}=\frac{(\text { Size of the targeted file })}{(\text { Total size of the answers })},
$$

and the optimal efficiency is derived as the supremum of the PIR rate, which is called the PIR capacity [18], and has been studied in many papers. The paper [19] derived the capacity of PIR with disjoint collusion patterns, which is a special case of the arbitrary collusion patterns. This model is extended to the arbitrary collusion patterns by [20], [21], [22], where PIR protocols are constructed on coded data storage. On the replicated data storage, the capacity of PIR with arbitrary collusion patterns [23] and the capacity of SPIR with arbitrary collusion and eavesdropping patterns [24] are characterized by the solution of a linear programming problem. For the PIR/SPIR with thresholds, i.e., $\mathfrak{A}(\mathfrak{B})$ consists of all subsets with cardinality at least $r$ (at most $t$ ), the papers [25], [26], and [27] derived the capacity of PIR with $t$ colluding and $b$ byzantine servers, the capacity of SPIR with $t$ colluding and e eavesdropping servers with an assumption on the shared randomness of the servers, and the PIR/SPIR capacity with $t$ colluding, e eavesdropping, $r$ responsive, and $b$ byzantine servers, under various assumptions on the protocols and the shared randomness, respectively. The paper [28] constructed PIR/SPIR protocols with $t$ colluding, $r$ responsive, and $b$ byzantine servers. However, no existing study discussed SPIR with arbitrary response and collusion patterns.

\section{B. Main Results}

As the first result, this paper shows that $(\mathfrak{A}, \mathfrak{B}, \mathrm{n}, \mathfrak{f})$-SPIR implies $(\mathfrak{A}, \mathfrak{B}, n)$-NSS. To state this result, we formally define $(\mathfrak{A}, \mathfrak{B}, n, f)$-SPIR and $(\mathfrak{A}, \mathfrak{B}, n)$-NSS protocols with incomplete security by the measures of correctness and secrecy. With abuse of notation, we prove that given an $(\mathfrak{A}, \mathfrak{B}, \mathrm{n}, \mathfrak{f})$-SPIR protocol with nearly complete security, we propose a method to construct an $(\mathfrak{A}, \mathfrak{B}, \mathrm{n})$-NSS protocol with nearly complete security. The proof idea is simply described as follows. To construct an $(\mathfrak{A}, \mathfrak{B}, n)$-NSS protocol from a given $(\mathfrak{A}, \mathfrak{B}, n, f)$ SPIR protocol, the dealer simulates the ( $\mathfrak{A}, \mathfrak{B}, \mathrm{n}, \mathrm{f})$-SPIR protocol while setting the secret of NSS as one of the files, and encodes the $\mathrm{n}$ answers as $\mathrm{n}$ shares. Then, the shares indexed by $\mathfrak{A}$ reconstruct the secret by the SPIR's correctness. For the secrecy part, we prove that the shares indexed by $\mathfrak{B}$ have no information of the secret from the SPIR's two secrecy conditions.

When the SPIR and NSS protocols have complete correctness and secrecy, these protocols are called completely secure and denoted by CSSPIR and CSNSS protocols, respectively. One interesting corollary of our first result is an upper bound on the CSSPIR capacity with arbitrary response and collusion patterns. Similar to the PIR rate defined in (1), the SS rate 11 $R_{\mathrm{SS}}$ is defined as

$$
R_{\mathrm{SS}}=\frac{(\text { Size of the secret })}{\text { (Total size of the shares) }} .
$$

In our conversion from SPIR to NSS, any SPIR protocol with PIR rate $R_{\text {PIR }}$ is converted into an NSS protocol with SS rate $R_{\mathrm{SS}}=R_{\text {PIR }}$. Furthermore, any $(\mathfrak{A}, \mathfrak{B}, \mathrm{n})$-CSNSS protocols satisfy $\mathrm{n} R_{\mathrm{SS}} \leq \delta(\mathfrak{A}, \mathfrak{B}):=\min \{|\mathcal{A}-\mathcal{B}| \mid \mathcal{A} \in \mathfrak{A}, \mathcal{B} \in \mathfrak{B}\}$ [12], [13], [14]. Thus, we obtain $\mathrm{n} R_{\mathrm{PIR}} \leq \delta(\mathfrak{A}, \mathfrak{B})$ for any $(\mathfrak{A}, \mathfrak{B}, \mathrm{n}, \mathfrak{f})$-CSSPIR protocols. This is the first result to characterize the CSSPIR capacity of arbitrary collusion and response patterns.

As a special case, when $\mathfrak{A}(\mathfrak{B})$ consists of all subsets of [n] with cardinality at least $r$ (at most $t$ ), we obtain $\delta(\mathfrak{A}, \mathfrak{B})=r-t$, i.e., the rate of $(\mathfrak{A}, \mathfrak{B}, \mathrm{n}, \mathrm{f})$-CSSPIR is upper bounded by $(r-t) / n$. This special case generalizes the result of Holzbaur et al. [27], which proved the same upper bound for a restricted class of CSSPIR, i.e., linear CSSPIR with additive randomness. Since the protocol by Tajeddine et al. [28] achieves this upper bound? our upper bound proves that the capacity of CSSPIR with $r$ responses and $t$ colluding servers is $(r-t) / n$.

Our second main result is the equivalence of linear $(\mathfrak{A}, \mathfrak{B}, \mathrm{n}, \mathrm{f})$-CSSPIR and linear $(\mathfrak{A}, \mathfrak{B}, \mathrm{n})$-CSNSS. Linear $(\mathfrak{A}, \mathfrak{B}, n, f)$-SPIR (linear $(\mathfrak{A}, \mathfrak{B}, n)$-NSS) is a well-known class of SPIR (NSS) in which the answers (shares) are generated by linear encoders. To prove this result, we define multitarget monotone span programs (MMSP) with general ac-

\footnotetext{
${ }^{1}$ The efficiency of SS is often considered with information rate and information ratio [32]. The information rate is defined by replacing the denominator of (2) by the maximum size of a share, and the information ratio is the inverse of the information rate. However, we define the SS rate for the correspondence with the PIR rate.

${ }^{2}$ In [28], the notation $r$ is for the number of unresponsive servers, which is $n-r$ in our paper. Moreover, [28] defined the denominator of the PIR rate (1) as "bit size of all responsive servers".
} 
cess structures $(\mathfrak{A}, \mathfrak{B})$, which we call $(\mathfrak{A}, \mathfrak{B}, \mathrm{n})-M M S P$. An MMSP is a pair of a matrix and a map with two linearalgebraic conditions. To prove the equivalence result, we separately prove that a $(\mathfrak{A}, \mathfrak{B}, n)$-CSNSS protocol, a $(\mathfrak{A}, \mathfrak{B}, n)$ MMSP, and a $(\mathfrak{A}, \mathfrak{B}, n)-C S S P I R$ protocol, are constructed from a $(\mathfrak{A}, \mathfrak{B}, n)$-CSSPIR protocol, a $(\mathfrak{A}, \mathfrak{B}, n)$-CSNSS protocol, and a $(\mathfrak{A}, \mathfrak{B}, n)$-MMSP, respectively, which implies the equivalence of the three protocols. Our equivalence result of $(\mathfrak{A}, \mathfrak{B}, n)$-CSNSS and ( $\mathfrak{A}, \mathfrak{B}, n)$-MMSP generalizes the equivalence of completely secure SS and monotone span program [29], [30], [31]. Since there exists a linear CSNSS protocol for any access structure $(\mathfrak{A}, \mathfrak{B})$ [16], the same existence holds for CSSPIR by our second result.

\section{Basic Idea in Equivalence}

The key intuition for the equivalence is the similarity in the structure of the protocols (see Figure 1 and Table \). From this similarity, the server secrecy of SPIR is closely related to the secrecy of NSS, while the user secrecy of SPIR is not covered in NSS. Thus, we can roughly state that SPIR is more complicated protocol than NSS protocol. With this idea, conversion from the complicated SPIR protocol to the simpler NSS protocol is obtained by utilizing only a part of the SPIR protocol as the NSS protocol. That is, we only need the operational properties of SPIR, rather than some algebraic structure, for converting it into NSS. As a result, the conversion from SPIR to NSS can be completed without any linearity assumption.

On the other hand, to construct conversion from NSS to SPIR, we need to add the user secrecy to the NSS protocol. This addition of user secrecy is hard to be implemented only with the operational definition of NSS protocols. Thus, instead, we convert linear NSS into linear SPIR. With the linearity algebraic structure in linear NSS, we add the user secrecy to NSS so that it operates the SPIR's tasks. As a result, the conversion from NSS to SPIR is limited to linear protocols in this paper.

One interesting observation of the conversion from NSS to SPIR is that the one message protocol, NSS, is evolved into the multiple message protocol, SPIR. This task is completed in our conversion from linear NSS to linear SPIR by the following idea. When $k$-th file $M_{k}$ is the desired file of the user, the user forces the servers to choose $M_{k}$ as a secret of the NSS without leaking $k$ and answer the generated shares of the NSS. This forcing step by the user is accomplished with a wellestablished query structure. Finally, the user reconstructs $M_{k}$ by collecting an authorized set of shares (answers).

\section{Organization}

The remainder of the paper is organized as follows. Section III gives the definitions of NSS, SPIR, and related concepts. Section III presents the main results of the paper. Section IV proves the conversions from SPIR to NSS and from linear SPIR to linear NSS. Sections $\nabla$ and $\nabla \mathrm{VI}$ prove the conversions from linear CSNSS to MMSP and from MMSP to linear CSSPIR, respectively. Section VII give two examples of the constructions: a construction with general non-threshold
TABLE II

DEFINITION OF SYMBOLS

\begin{tabular}{|c|c|c|}
\hline Symbol & SPIR & Secret Sharing \\
\hline \hline $\mathrm{n}$ & Number of servers & Number of shares \\
\hline $\mathrm{f}$ & Number of files & - \\
\hline $\mathrm{m}$ & Size of one file & Size of secret \\
\hline $\mathrm{r}$ & Number of responsive servers & Reconstruction threshold \\
\hline $\mathrm{t}$ & Number of colluding servers & Secrecy threshold \\
\hline
\end{tabular}

access structure, and optimal constructions of linear CSNSS and linear CSSPIR when the access structure with thresholds. Section VIII is the conclusion of the paper.

Notations: We denote random variables by uppercase letters (e.g., $A, B$ ), and values of the random variables by lowercase letters (e.g., $a, b$ ), sets by calligraphy letters (e.g., $\mathcal{A}, \mathcal{B}$ ), parameters in protocols by sans serif lowercase letters (e.g., a,b), and matrices by sans serif upper letters (e.g., A, B). We also denote $[n, m]=\{n, n+1, \ldots, m\}$ and $[n]=[1: n]$. For any set or sequence $A=\left\{A_{1}, \ldots, A_{n}\right\}$ and any $\mathcal{X} \subset[n]$, we denote $A_{X}:=\left\{A_{i} \mid i \in \mathcal{X}\right\}$. For any $n \times m$ matrix $\mathrm{A}$ and any $\mathcal{X} \subset[n]$, we denote $A_{\mathcal{X}}$ is the restricted matrix by the rows indexed by $\mathcal{X}$. The finite field of order $q$ is denoted by $\mathbb{F}_{q}$. For a set $\mathcal{A}, \operatorname{id}_{\mathcal{A}}$ denotes the identity map on $\mathcal{A}$. For a random variable $X, \operatorname{Pr}_{X}[f(X)]$ is the probability that $X$ satisfies the condition $f(X)$.

\section{FORMAL DEFINITION OF NSS AND SPIR}

In this section, we formally define SPIR, NSS, and MMSP. For these definitions, we first define access structures.

Definition 1 (Access structure). Let $\mathrm{n}$ be a positive integer. We call $\mathfrak{A} \subset 2^{[\mathrm{n}]}$ an monotone increasing collection if $\mathcal{A} \in \mathfrak{A}$ implies $C \in \mathfrak{A}$ for any $\mathcal{A} \subset \mathcal{C} \subset$ [n]. In contrast, we call $\mathfrak{B} \subset 2^{[\mathrm{n}]}$ a monotone decreasing collection if $\mathcal{B} \in \mathfrak{B}$ implies $C \in \mathfrak{B}$ for any $C \subset \mathcal{B}$. An access structure on $[\mathrm{n}]$ is defined as a pair of monotone increasing and decreasing collections $\mathfrak{A}$ and $\mathfrak{B} \subset 2^{[\mathrm{n}]}$ such that $\mathfrak{A} \cap \mathfrak{B}=\emptyset$.

Example 1. When $\mathrm{n}=3, \mathfrak{A}=\{\{2,3\},\{1,2,3\}\}$, and $\mathfrak{B}=\{\emptyset,\{1\},\{2\},\{3\},\{1,2\}\}$, the pair $(\mathfrak{A}, \mathfrak{B})$ forms an access structure. The monotone increasing collection $\mathfrak{A}$ consists of all subsets containing $\{2,3\}$, and the monotone decreasing collection $\mathfrak{B}$ consists of all subsets contained in $\{1,2\}$ or $\{3\}$. In this example, the subsets of size 2 are contained in different collections: $\{2,3\} \in \mathfrak{A},\{1,2\} \in \mathfrak{B}$, and $\{1,3\} \notin \mathfrak{A} \cup \mathfrak{B}$.

\section{A. Formal definition of symmetric private information re- trieval (SPIR)}

We formally define a SPIR protocol with one user and $n$ servers as follows.

Definition 2 (( $\mathfrak{A}, \mathfrak{B}, \mathrm{n}, \mathrm{f})$-SPIR). Files are given as a uniform random variable $M=\left(M_{1}, \ldots, M_{\mathrm{f}}\right) \in \mathcal{M}^{\dagger}$ and $\mathrm{m}:=|\mathcal{M}|$. Each of $\mathrm{n}$ servers contains the files $M$. Let $U_{\mathrm{SPIR}} \in \mathcal{U}_{\mathrm{SPIR}}$ be uniform random variable, called the random seed for servers, and the random seed $U_{\text {SPIR }}$ is encoded as $h\left(U_{\mathrm{SPIR}}\right)=T=$ $\left(T_{1}, \ldots, T_{n}\right) \in \mathcal{T} \subset \mathcal{T}_{1} \times \cdots \times \mathcal{T}_{\mathrm{n}}$ by the shared randomness encoder $h$. The randomness $T$ is distributed so that $j$-th server 
contains $T_{j}$. Let $K$ be a uniform random variable in [f], which represents the user's input. When $K=k$, the targeted file is $M_{k}$.

A protocol $\Phi_{\mathrm{SPIR}}^{\mathrm{m}}$ is defined by the deterministic mappings $f, g_{1}, \ldots, g_{\mathrm{n}}$ in the following steps.

- Query: Depending on the user's private uniform randomness $R \in \mathcal{R}$, the user prepares $\mathrm{n}$ queries by

$$
Q=\left(Q_{1}, \ldots, Q_{\mathrm{n}}\right)=f(K, R) \in Q_{1} \times \cdots \times Q_{\mathrm{n}}
$$

and sends the $j$-th query $Q_{j}$ to the $j$-th server.

- Answer: The j-th server returns

$$
D_{j}=g_{j}\left(Q_{j}, M, T_{j}\right) \in \mathcal{D}_{j}
$$

to the user.

For an access structure $(\mathfrak{A}, \mathfrak{B})$ on $[\mathrm{n}]$. the protocol $\Phi_{\mathrm{SPIR}}^{\mathrm{m}}$ is called an $(\mathfrak{A}, \mathfrak{B}, \mathrm{n}, \mathrm{f})$-SPIR protocol with security $(\alpha, \beta, \gamma)$ if the following conditions are satisfied.

- Correctness: We define the user's maximum likelihood decoder

$$
\hat{m}_{d_{\mathcal{A}, r, k}}^{\mathrm{ML}}:=\underset{m_{k} \in \mathcal{M}}{\operatorname{argmax}} \operatorname{Pr}\left[D_{\mathcal{A}}=d_{\mathcal{A}} \mid\left(M_{K}, R, K\right)=\left(m_{k}, r, k\right)\right] .
$$

Then,

$$
\alpha \geq \alpha\left(\Phi_{\mathrm{SPIR}}^{\mathrm{m}}\right):=\max _{r \in \mathcal{R}, k \in[\mathrm{f}], \mathcal{A} \in \mathfrak{A}} \operatorname{Pr}_{M_{k}, D_{\mathcal{A}}}\left[M_{k} \neq \hat{m}_{D_{\mathcal{A}}, r, k}^{\mathrm{ML}}\right] .
$$

\section{- Server secrecy:}

$$
\beta \geq \beta\left(\Phi_{\mathrm{SPIR}}^{\mathrm{m}}\right):=\max _{r \in \mathcal{R}, k \in[\mathrm{f}]} I\left(D ; M_{[\mathrm{f}] \backslash\{k\}} \mid R=r, K=k\right) .
$$

\section{- User secrecy.}

$$
\gamma \geq \gamma\left(\Phi_{\mathrm{SPIR}}^{\mathrm{m}}\right):=\max _{\mathcal{B} \in \mathcal{B}} I\left(K ; Q_{\mathcal{B}}\right)
$$

The PIR rate of the protocol $\Phi_{\mathrm{SPIR}}^{\mathrm{m}}$ is defined as

$$
R_{\mathrm{PIR}}\left(\Phi_{\mathrm{SPIR}}^{\mathrm{m}}\right):=\frac{\log \mathrm{m}}{\sum_{j=1}^{\mathrm{n}} \log \left|\mathcal{D}_{j}\right|} .
$$

The shared randomness rate is defined as

$$
R_{U_{\mathrm{SPIR}}}=\frac{\log \left|\mathcal{U}_{\mathrm{SPIR}}\right|}{\log \mathrm{m}} .
$$

Remark 1. From the definition of the protocol, it seems natural to define the server secrecy condition as $\beta^{\prime}\left(\Phi_{\mathrm{SPIR}}^{\mathrm{m}}\right):=$ $\max _{\mathcal{A} \in \mathfrak{A}, r \in \mathcal{R}, k \in[\mathrm{f}]} I\left(D_{\mathcal{A}} ; M_{[\mathrm{f}] \backslash\{k\}} \mid R=r, K=k\right)$, which is the maximization of the $\beta^{\prime}\left(\Phi_{\text {SPIR }}^{\mathrm{m}}\right)$ defined in (6). Indeed, we have $\beta^{\prime}\left(\Phi_{\text {SPIR }}^{\mathrm{m}}\right)=\beta\left(\Phi_{\text {SPIR }}^{\mathrm{m}}\right)$ since the collection $\mathfrak{A}$ contains the set $[\mathrm{n}]$ by the monotone increasing property.

In the above security conditions, $\alpha\left(\Phi_{\text {SPIR }}^{\mathrm{m}}\right)$ is the worstcase error probability with the maximum likelihood decoder, $\beta\left(\Phi_{\text {SPIR }}^{\mathrm{m}}\right)$ is the worst-case leakage of the non-targeted files to the user, and $\gamma\left(\Phi_{\text {SPIR }}^{\mathrm{m}}\right)$ is the worst-case leakage of the index $K$ to the colluding servers. If $(\alpha, \beta, \gamma)=(0,0,0)$, the $(\mathfrak{A}, \mathfrak{B}, \mathrm{n}, \mathrm{f})$-SPIR protocol has complete security.

Definition 3 (CSSPIR). A ( $\mathfrak{A}, \mathfrak{B}, \mathrm{n}, \mathrm{f})-S P I R$ protocol with security $(\alpha, \beta, \gamma)=(0,0,0)$ is called a completely secure $(\mathfrak{A}, \mathfrak{B}, \mathrm{n}, \mathrm{f})$-SPIR $((\mathfrak{A}, \mathfrak{B}, \mathrm{n}, \mathrm{f})$-CSSPIR) protocol.
In a $(\mathfrak{A}, \mathfrak{B}, \mathrm{n}, \mathrm{f})$-CSSPIR protocol, the user can recover the targeted file $M_{K}$ without error from the answers indexed by any $\mathcal{A} \in \mathfrak{A}$, but the servers indexed by $\mathcal{B} \in \mathfrak{B}$ obtain no information of $K$. The achievable rate and capacity of $(\mathfrak{A}, \mathfrak{B}, \mathrm{n}, \mathrm{f})$-CSSPIR are defined as follows.

Definition 4 (Achievability of ( $\mathfrak{A}, \mathfrak{B}, \mathrm{n}, \mathrm{f}$ )-CSSPIR). A PIR rate $R$ is achievable if there is a sequence of $(\mathfrak{A}, \mathfrak{B}, \mathrm{n}, \mathrm{f})$ CSSPIR protocols $\left\{\Phi_{\mathrm{SPIR}}^{\mathrm{m}}\right\}_{\mathrm{m} \in \mathbb{N}}$ such that $R_{\mathrm{PIR}}\left(\Phi_{\mathrm{SPIR}}^{\mathrm{m}}\right) \rightarrow R$ as $\mathrm{m} \rightarrow \infty$.

Definition 5 (Capacity of ( $\mathfrak{A}, \mathfrak{B}, \mathrm{n}, \mathrm{f})$-CSSPIR). Capacity of $(\mathfrak{A}, \mathfrak{B}, \mathrm{n}, \mathrm{f})-C S S P I R, C_{\mathrm{SPIR}}^{(\mathfrak{H}, \mathfrak{B}, \mathrm{f})}$, is the supremum of achievable rates of $(\mathfrak{A}, \mathfrak{B}, \mathrm{n}, \mathrm{f})-C S S P I R$.

As special cases, SPIR with thresholds and linear SPIR are defined as follows.

Definition $6((\mathrm{r}, \mathrm{t}, \mathrm{n}, \mathrm{f})-\mathrm{SPIR})$. When $\mathfrak{A}=\{\mathcal{A} \subset[\mathrm{n}]|| \mathcal{A} \mid \geq$ r) and $\mathfrak{B}=\{\mathcal{B} \subset[\mathrm{n}]|| \mathcal{B} \mid \leq \mathrm{t}\}$, ( $\mathfrak{A}, \mathfrak{B}, \mathrm{n}, \mathrm{f})$-SPIR protocols are called $(\mathrm{r}, \mathrm{t}, \mathrm{n}, \mathrm{f})-S P I R$ protocols.

Definition 7 (Linear SPIR). A protocol $\Phi_{\mathrm{SPIR}}^{\mathrm{m}}$ is called a linear SPIR protocol if the following conditions are satisfied with a map $\tau:[\mathrm{z}] \rightarrow[\mathrm{n}]$, called the position map.

Vector representation of files The files $M_{i}$ are written as a vector in $\mathbb{F}_{q}^{\mathrm{x}}$. The entire file is written by the concatenated vector $M=\left(M_{1}, \ldots, M_{\mathrm{f}}\right) \in \mathbb{F}_{q}^{\mathrm{fx}}$.

Linearity of shared randomness The random seed $U_{\text {SPIR }}$ is written by a uniform random vector in $\mathbb{F}_{q}^{\mathrm{y}}$. The randomness encoder is written as a matrix $\mathrm{H} \in \mathbb{F}_{q}^{\mathrm{z} \times \mathrm{y}}$ and the shared randomness is written as $T=\mathrm{H} U_{\mathrm{SPIR}} \in \mathbb{F}_{q}^{\mathrm{Z}}$. The randomness of the $j$-th server is written as $T_{j}=$ $\mathrm{H}_{\tau^{-1}(j)} T^{\prime} \in \mathbb{F}_{q}^{\left|\tau^{-1}(j)\right|}$.

Linearity of servers The answer of the $j$-th server $D_{j}$ is written as the sum of the shared randomness $T_{j}$ and the encoded output of the files $M$ by the linear function $Q_{\tau^{-1}(j)}$, which depends on the query, i.e.,

$$
D_{j}=Q_{\tau^{-1}(j)} M+T_{j} \in \mathbb{F}_{q}^{\left|\tau^{-1}(j)\right|} .
$$

Therefore, we can consider that the query to the $j$-th server is given as the linear function, a matrix, $Q_{\tau^{-1}(j)} \in$ $\mathbb{F}_{q}^{z \times f x}$.

A linear SPIR is described by the triplet $(\mathrm{H}, Q, \tau)$ of randomness encoder $\mathrm{H} \in \mathbb{F}_{q}^{z \times y}$, random matrix of query $Q \in \mathbb{F}_{q}^{\mathrm{Zxfx}}$, and position map $\tau:[\mathrm{z}] \rightarrow[\mathrm{n}]$. Due to the above conditions, the PIR rate and the shared randomness rate of a linear SPIR protocol are $\mathrm{x} / \mathrm{z}$ and $\mathrm{y} / \mathrm{x}$, respectively.

Remark 2. Our definition of linear SPIR generalizes the definition of Holzbaur et al. [27]. The linear SPIR defined in [27] corresponds to our definition with $\left|\tau^{-1}(j)\right|=c$ for a fixed $c$.

In the definition of linear PIR, linearity is required for the generation of the shared randomness and the servers' answers. We define a new subclass of linear PIR with a specific linear condition on the user's encoding as follows. 
Definition 8 (Projected linear SPIR). A linear SPIR protocol $\Phi_{\text {SPIR }}^{\mathrm{m}}$ is called a projected linear SPIR protocol if the query $Q=\left(Q_{\tau^{-1}(1)}, \ldots, Q_{\tau^{-1}(\mathrm{n})}\right)$ satisfies the following condition;

Linearity of user The uniform randomness $R$ of the user is written as a matrix in $\mathbb{F}_{q}^{\mathrm{y} \times \mathrm{fx}}$. The query is written as

$$
Q=\mathrm{JE}_{k}+\mathrm{H} R \in \mathbb{F}_{q}^{\mathrm{zxfx}},
$$

where $\mathrm{J}$ is a matrix in $\mathbb{F}_{q}^{\mathrm{Z} \times \mathrm{x}}, \mathrm{E}_{k} \in \mathbb{F}_{q}^{\mathrm{X} \times \mathrm{fx}}=\mathbb{F}_{q}^{\mathrm{X} \times \mathrm{x}} \times \cdots \times \mathbb{F}_{q}^{\mathrm{X} \times \mathrm{X}}$ is the matrix whose $k$-th block is the $\mathrm{x} \times \mathrm{x}$ identity matrix and the other blocks are zero, and $\mathrm{H} \in \mathbb{F}_{q}^{\mathrm{Zxy}}$ is the same matrix as the shared randomness encoder. The query to the $j$-th server is written as $Q_{\tau^{-1}(j)} \in \mathbb{F}_{q}^{\left|\tau^{-1}(j)\right| \times \mathrm{fx}}$.

A projected linear SPIR is described by the triplet $(\mathrm{H}, \mathrm{J}, \tau)$ of randomness encoder $\mathrm{H} \in \mathbb{F}_{q}^{Z \times y}$, user's query encoder $\mathrm{J} \in$ $\mathbb{F}_{q}^{\mathrm{z} \times \mathrm{x}}$, and position map $\tau:[\mathrm{z}] \rightarrow[\mathrm{n}]$.

Whereas the size of the query $Q \in \mathbb{F}_{q}^{\mathrm{zxfx}}$ in linear SPIR is increasing with the number of files $f$, that of projected linear SPIR $\mathrm{J} \in \mathbb{F}_{q}^{\mathrm{Zx}}$ is smaller and independent of the size of the files.

We define a projection $\hat{p}$ from linear SPIR protocols into projected linear SPIR protocols as follows.

Definition 9 (Projection). For a linear SPIR protocol $\Phi_{\text {SPIR' }}^{\mathrm{m}}$, let $\mathrm{Q}=\left(\mathrm{Q}^{\prime}, \mathrm{Q}^{\prime \prime}\right) \in \mathbb{F}_{q}^{\mathrm{Z} \times \mathrm{x}} \times \mathbb{F}_{q}^{\mathrm{Z} \times(\mathrm{f}-1) \mathrm{x}}$ be the matrix of query when $K=1$ and $R=0$. Further, let $\mathrm{H} \in \mathbb{F}_{q}^{\mathrm{zxy}}$ be the randomness encoder and $\tau$ be the position map of $\Phi_{\mathrm{SPIR}}^{\mathrm{m}}$. Then, we define a map $\hat{p}$ that maps $\Phi_{\mathrm{SPIR}}^{\mathrm{m}}$ to the projected linear SPIR protocol described by the triplet $\left(\mathrm{H}, \mathrm{Q}^{\prime}, \tau\right)$ of the randomness encoder, user's query encoder, and position map. The map $\hat{p}$ is a projection to projected linear SPIR because any projected linear SPIR protocol is mapped to itself.

\section{B. Formal definition of non-perfect secret sharing (NSS)}

We formally define an NSS protocol with one dealer and $n$ players as follows.

Definition 10 (( $\mathfrak{A}, \mathfrak{B}, \mathrm{n})$-NSS). A secret is given as a uniform random variable $L \in \mathcal{M}$ and $\mathrm{m}:=|\mathcal{M}|$. A protocol $\Phi_{\mathrm{NSS}}^{\mathrm{m}}$ is defined by the following deterministic map (the encoding map) $f$ to generate $n$ shares in the share generation step;

- Share generation: Depending on the dealer's private uniform randomness $U_{\mathrm{NSS}} \in \mathcal{U}_{\mathrm{NSS}}$, the dealer prepares $\mathrm{n}$ shares by

$$
S=\left(S_{1}, \ldots, S_{\mathrm{n}}\right)=f\left(L, U_{\mathrm{NSS}}\right) \in \mathcal{S}_{1} \times \cdots \times \mathcal{S}_{\mathrm{n}},
$$

and sends the $j$-th share $S_{j}$ to the $j$-th player.

For an access structure $(\mathfrak{A}, \mathfrak{B})$ on $[\mathrm{n}]$, the protocol $\Phi_{\mathrm{NSS}}^{\mathrm{m}}$ is called an $(\mathfrak{A}, \mathfrak{B}, \mathrm{n})-N S S$ protocol with security $(\alpha, \beta)$ if the following conditions are satisfied.

- Correctness: We define the maximum likelihood decoder

$$
\hat{\ell}_{s_{\mathcal{A}}}^{\mathrm{ML}}:=\underset{\ell \in \mathcal{M}}{\operatorname{argmax}} \operatorname{Pr}\left[S_{\mathcal{A}}=s_{\mathcal{A}} \mid L=\ell\right] .
$$

Then,

$$
\alpha \geq \alpha\left(\Phi_{\mathrm{NSS}}^{\mathrm{m}}\right):=\max _{\mathcal{A} \in \mathfrak{A}} \operatorname{Pr}_{L, S_{\mathcal{A}}}\left[L \neq \hat{\ell}_{S_{\mathcal{H}}}^{\mathrm{ML}}\right]
$$

- Secrecy:

$$
\beta \geq \beta\left(\Phi_{\mathrm{NSS}}^{\mathrm{m}}\right):=\max _{\mathcal{B} \in \mathcal{B}} I\left(L ; S_{\mathcal{B}}\right) .
$$

The SS rate of the protocol $\Phi_{\mathrm{NSS}}^{\mathrm{m}}$ is defined as

$$
R_{\mathrm{SS}}\left(\Phi_{\mathrm{NSS}}^{\mathrm{m}}\right):=\frac{\log \mathrm{m}}{\sum_{j=1}^{\mathrm{n}} \log \left|\mathcal{S}_{j}\right|} .
$$

The randomness rate is defined as

$$
R_{U_{\mathrm{NSS}}}=\frac{\log \left|\mathcal{U}_{\mathrm{NSS}}\right|}{\log \mathrm{m}}
$$

In the above security conditions, $\alpha\left(\Phi_{\mathrm{SPIR}}^{\mathrm{m}}\right)$ is the worstcase error probability with the maximum likelihood decoder and $\beta\left(\Phi_{\mathrm{SPIR}}^{\mathrm{m}}\right)$ is the worst-case leakage of the secret to the forbidden set of shares. If $(\alpha, \beta)=(0,0)$, the $(\mathfrak{A}, \mathfrak{B}, \mathrm{n})$ CSNSS protocol has complete security.

Definition 11 (CSNSS). A ( $\mathfrak{A}, \mathfrak{B}, \mathrm{n})$-NSS protocol with security $(\alpha, \beta)=(0,0)$ is called a completely secure $(\mathfrak{A}, \mathfrak{B}, \mathrm{n})-N S S$ $((\mathfrak{U}, \mathfrak{B}, \mathrm{n})$-CSNSS) protocol.

In a $(\mathfrak{A}, \mathfrak{B}, n)$-CSNSS protocol, the players indexed by any $\mathcal{A} \in \mathfrak{A}$ can recover $L$ without error, but the players indexed by any $\mathcal{B} \in \mathfrak{B}$ obtain no information of $L$. The achievable rate and capacity of $(\mathfrak{A}, \mathfrak{B}, n)$-CSNSS are defined as follows.

Definition 12 (Achievability of ( $\mathfrak{A}, \mathfrak{B}, \mathrm{n}$ )-CSNSS). A SS rate $R$ is achievable if there is a sequence of $(\mathfrak{A}, \mathfrak{B}, \mathrm{n})-C S N S S$ protocols $\left\{\Phi_{\mathrm{NSS}}^{\mathrm{m}}\right\}_{\mathrm{m} \in \mathbb{N}}$ such that $R_{\mathrm{SS}}\left(\Phi_{\mathrm{NSS}}^{\mathrm{m}}\right) \rightarrow R$ as $\mathrm{m} \rightarrow \infty$.

Definition 13 (Capacity of ( $\mathfrak{A}, \mathfrak{B}, \mathrm{n})$-CSNSS). Capacity of $(\mathfrak{A}, \mathfrak{B}, \mathrm{n})-C S N S S, C_{\mathrm{NSS}}^{(\mathfrak{A}, \mathfrak{n})}$, is the supremum of achievable rates of $(\mathfrak{A}, \mathfrak{B}, \mathrm{n})-C S N S S$.

Remark 3. When $\mathfrak{A} \cup \mathfrak{B}=2^{[n]}$, the protocol in Definition 10 is called a (perfect) secret sharing protocol. Since all results in this paper are obtained for any general access structure, all results also apply to perfect SS.

As special cases, NSS with thresholds and linear NSS are defined as follows.

Definition $14((\mathrm{r}, \mathrm{t}, \mathrm{n})$-NSS). When $\mathfrak{A}=\{\mathcal{A} \subset[\mathrm{n}]|| \mathcal{A} \mid \geq \mathrm{r}\}$ and $\mathfrak{B}=\{\mathcal{B} \subset[\mathrm{n}]|| \mathcal{B} \mid \leq \mathrm{t}\},(\mathfrak{A}, \mathfrak{B}, \mathrm{n})-N S S$ protocols are called $(\mathrm{r}, \mathrm{t}, \mathrm{n})-N S S$ protocols.

Definition 15 (Linear NSS). A protocol $\Phi_{\mathrm{NSS}}^{\mathrm{m}}$ is called a linear NSS protocol if the encoding map $f$ satisfies the following conditions with a position map $\tau:[\mathrm{z}] \rightarrow[\mathrm{n}]$.

Vector representation of secret The secret $L$ is written as a vector in $\mathbb{F}_{q}^{\mathrm{X}}$.

Vector representation of randomness The dealer's private randomness $U_{\mathrm{NSS}}$ is written as a uniform random vector in $\mathbb{F}_{q}^{\mathrm{y}}$.

Linearity of share generation The encoder $f$ is written as a linear map from $\mathbb{F}_{q}^{\mathrm{x}+\mathrm{y}}$ to $\mathbb{F}_{q}^{\mathrm{Z}}$ that maps $\left(Z_{1}, \ldots, Z_{\mathrm{z}}\right)=$ $f\left(L, U_{\mathrm{NSS}}\right)$. The $j$-th secret is written as $S_{j}=\left(Z_{i} \mid i \in\right.$ $\left.\tau^{-1}(j)\right) \in \mathbb{F}_{q}^{\left|\tau^{-1}(j)\right|}$

Thus, a linear NSS $\Phi_{\mathrm{NSS}}^{\mathrm{m}}$ is described by the pair $(f, \tau)$ of a linear map $f: \mathbb{F}_{q}^{\mathrm{x}+\mathrm{y}} \rightarrow \mathbb{F}_{q}^{\mathrm{z}}$ and a position map $\tau:[\mathrm{z}] \rightarrow[\mathrm{n}]$. Due to the above conditions, the SS rate and randomness rate of the linear NSS are $\mathrm{x} / \mathrm{z}$ and $\mathrm{y} / \mathrm{x}$, respectively. 
C. Formal definition of multi-target monotone span program (MMSP)

We define a multi-target monotone program (MMSP) with an access structure as follows.

Definition 16 (Multi-target monotone span program (MMSP)). Given positive integers $\mathrm{n}, \mathrm{x}, \mathrm{y}, \mathrm{z}$, a pair $\mathcal{P}=(\mathrm{G}, \tau)$ of a matrix $\mathrm{G}=\left(\mathrm{G}^{\prime}, \mathrm{G}^{\prime \prime}\right) \in \mathbb{F}_{q}^{\mathrm{z} \times(\mathrm{x}+\mathrm{y})}=\mathbb{F}_{q}^{\mathrm{z} \times \mathrm{x}} \times \mathbb{F}_{q}^{\mathrm{z} \times \mathrm{y}}$ and a position map $\tau:[\mathrm{z}] \rightarrow[\mathrm{n}]$ is called a multi-target monotone span program (MMSP).

Let $\mathbf{e}_{i} \in \mathbb{F}_{q}^{\mathrm{x}+\mathrm{y}}$ be the row vector with 1 in the $i$-th coordinate and 0 in the others. Let $\mathcal{E}:=\left\{\mathbf{e}_{1}, \ldots, \mathbf{e}_{\mathrm{x}}\right\}$ and we say the following;

- $\mathcal{P}$ accepts $\mathfrak{A}$ if $\mathcal{E}$ is included in the row space of $\mathrm{G}_{\tau^{-1}(\mathcal{A})}$ for any $\mathcal{A} \in \mathfrak{A}$, and

- $\mathcal{P}$ rejects $\mathfrak{B}$ if $\operatorname{span} \mathcal{E} \cap$ rowspan $\mathrm{G}_{\tau^{-1}(\mathcal{B})}=\{0\}$ for any $\mathcal{B} \in \mathfrak{B}$.

An MMSP $\mathcal{P}$ is called $(\mathfrak{A}, \mathfrak{B}, \mathrm{n})-M M S P$ if $\mathcal{P}$ accepts $\mathfrak{A}$ and rejects $\mathfrak{B}$. The MMSP rate $R_{\mathrm{MMSP}}$ is defined as the ratio $\mathrm{x} / \mathrm{Z}$.

Remark 4. Our definition of MMSP generalizes the definition in [32], [33], [34]. The MMSP defined in [32], [33], [34] corresponds to our definition with $\mathfrak{A} \cup \mathfrak{B}=2^{[n]}$ and $\mathfrak{A} \cap \mathfrak{B}=\emptyset$, i.e., every subset of $[n]$ is either authorized or forbidden. Our definition of MMSP also generalizes the monotone span programs [30], which corresponds to the case $\mathrm{x}=1$ and $\mathfrak{A} \cup \mathfrak{B}=2^{[\mathrm{n}]}$ for our MMSP definition.

The papers [29], [30], [31] proved the equivalence of linear perfect SS protocols with complete security and monotone span programs. This paper generalizes this relation to the equivalence of linear CSNSS protocols and MMSPs.

As special cases, we define $(\mathfrak{A}, \mathfrak{B}, n)$-MMSPs with thresholds as follows.

Definition $17((\mathrm{r}, \mathrm{t}, \mathrm{n})-\mathrm{MMSP})$. When $\mathfrak{A}=\{\mathcal{A} \subset[\mathrm{n}]|| \mathcal{A} \mid \geq$ $r\}$ and $\mathfrak{B}=\{\mathcal{B} \subset[\mathrm{n}]|| \mathcal{B} \mid \leq \mathrm{t}\}$, ( $\mathfrak{A}, \mathfrak{B}, \mathrm{n})$-MMSPs are called $(\mathrm{r}, \mathrm{t}, \mathrm{n})-M M S P s$.

$(r, t, n)$-MMSPs are related with maximum distance separable (MDS) codes. The column space of a matrix $A \in \mathbb{F}_{q}^{n \times k}$ is called an $(n, k)$-MDS code if any $k$ rows of $A$ are linearly independent. We give the relation between $(r, t, n)$-MMSPs and MDS codes by the following theorem.

Theorem 1. Let $\mathrm{G}=\left(\mathrm{G}^{\prime}, \mathrm{G}^{\prime \prime}\right) \in \mathbb{F}_{q}^{\mathrm{n} \times(\mathrm{r}-\mathrm{t})} \times \mathbb{F}_{q}^{\mathrm{n} \times \mathrm{t}}$. The following conditions are equivalent.

(a) With $\tau:=\mathrm{id}_{[\mathrm{n}]}$ and $(\mathrm{x}, \mathrm{y}, \mathrm{z}):=(\mathrm{r}-\mathrm{t}, \mathrm{t}, \mathrm{n}), \mathcal{P}=(\mathrm{G}, \tau)$ is an $(\mathrm{r}, \mathrm{t}, \mathrm{n})-M M S P$.

(b) $\mathrm{G}\left(\mathrm{G}^{\prime \prime}\right)$ is the generator matrix of an $(\mathrm{n}, \mathrm{r})-M D S$ code $((\mathrm{n}, \mathrm{t})-M D S$ code $)$.

Theorem 1 will be proved in Appendix A

The matrices $G$ and $G^{\prime \prime}$ with condition (b) of Theorem 1 have been applied for private information retrieval [40], [41], [42], [56], [28], secret sharing [2], [11], [35], [36], [37], [38], [39], wiretap channel II [43], [44], secure network coding [45], [46], [47], [48], [49], [50], [51], distributed storage system [52] and cryptography [53], [54]. Especially, Ozarow and Wyner's optimal wiretap channel code corresponds to the case $r=n$ and Shamir's secret sharing protocol corresponds to the case where $r=t+1$. Thus, from Theorem 1, $(r, t, n)$-MMSP with condition (a) characterizes the structure of those protocols and $(\mathfrak{A}, \mathfrak{B}, n)$-MMSP is a generalization of those protocols with general access structures.

\section{MAIN RESUltS}

In this section, we present our main theorems in three subsections. The subsections present, respectively, the conversions from SPIR to NSS, from linear CSNSS to MMSP, and from MMSP to projected linear CSSPIR.

\section{A. Conversion from SPIR to NSS}

The following protocol converts a SPIR protocol to an NSS protocol.

Protocol 1. Given a SPIR protocol $\Phi_{\mathrm{SPIR}}^{\mathrm{m}}$, an NSS protocol $\hat{\Phi}_{\mathrm{NSS}}^{\mathrm{m}}\left[\Phi_{\mathrm{SPIR}}^{\mathrm{m}}\right]$ is constructed by generating $\mathrm{n}$ shares as follows. Recall that from the definition of the SPIR protocol $\Phi_{\mathrm{SPIR}}^{\mathrm{m}}$, the symbols $\mathcal{M}, \mathcal{U}_{\mathrm{SPIR}}$, and $\mathcal{R}$ denote the space of files, random seeds for shared randomness, and private randomnesses of the user, respectively.

We define $r^{*} \in \mathcal{R}$ and $m_{[2: \mathrm{f}]}^{*} \in \mathcal{M}^{\mathrm{f}-1}$ as

$$
\begin{aligned}
& r^{*} \in \underset{r \in \mathcal{R}}{\operatorname{argmin}}\left\{\max _{\mathcal{B} \in \mathcal{B}} I\left(M_{1} ; D_{\mathcal{B}} \mid R=r, K=1\right)\right\} \\
& m_{[2: \mathrm{f}]}^{*} \in \underset{m_{[2: \mathrm{f}]} \in \mathcal{M}^{\mathrm{f}-1}}{\operatorname{argmin}} I\left(M_{1} ; D_{\mathcal{B}} \mid M_{[2: \mathrm{f}]}=m_{[2: \mathrm{f}]}, R=r^{*}, K=1\right) .
\end{aligned}
$$

The values $r^{*}$ and $m_{[2: \mathrm{f}]}^{*}$ are publicly known.

The dealer chooses the secret $L \in \mathcal{M}$ and the uniform randomness $U_{\mathrm{NSS}} \in \mathcal{U}_{\mathrm{SPIR}}$. The dealer simulates $\Phi_{\mathrm{SPIR}}^{\mathrm{m}}$ with $K:=1, M_{1}:=L$, and $U_{\mathrm{SPIR}}:=U_{\mathrm{NSS}}$ while fixing the user's private randomness as $R:=r^{*}$ and the $2 n d, \ldots, \mathrm{f}$-th file as $M_{[2: \mathrm{f}]}:=m_{[2: \mathrm{f}]}^{*}$. From this simulation of $\Phi_{\mathrm{SPIR}}^{\mathrm{m}}$, the dealer generates the answers $D_{1}, \ldots, D_{\mathrm{n}}$ of the SPIR protocol $\Phi_{\mathrm{SPIR}}^{\mathrm{m}}$ and sets the $j$-th share for NSS as $S_{j}:=D_{j}$.

Our first result is as follows.

Theorem 2. Let $\Phi_{\text {SPIR }}^{\mathrm{m}}$ be an $(\mathfrak{A}, \mathfrak{B}, \mathrm{n}, \mathrm{f})$-SPIR protocol with rate $R_{\mathrm{PIR}}$, shared randomness rate $R_{U_{\mathrm{SPIR}}}$, security $(\alpha, \beta, \gamma)$. Then, the NSS protocol $\hat{\Phi}_{\mathrm{NSS}}^{\mathrm{m}}\left[\Phi_{\mathrm{SPIR}}^{\mathrm{m}}\right]$ defined in Protocol 1 is an $(\mathfrak{A}, \mathfrak{B}, \mathrm{n})-N S S$ protocol $\Phi_{\mathrm{NSS}}^{\mathrm{m}}$ with $S S$ rate $R_{\mathrm{SS}}=R_{\mathrm{PIR}}$, randomness rate $R_{U_{\mathrm{NSS}}}=R_{U_{\mathrm{SPIR}}}$, and security $(\alpha, \xi(\alpha, \beta, \gamma))$, where

$$
\begin{aligned}
\xi(\alpha, \beta, \gamma)= & 2 \beta+(1-\alpha+4 \sqrt{2 \gamma \mathrm{f}}) \log \mathrm{m} \\
& +2 h_{2}(\sqrt{2 \gamma \mathrm{f}})+h_{2}(1-\alpha)+\log \alpha
\end{aligned}
$$

and $h_{2}$ is the binary entropy function $h_{2}(p)=-p \log p-$ $(1-p) \log (1-p)$. Here, $\xi(\alpha, \beta, \gamma)$ goes to 0 as $(\alpha, \beta, \gamma) \rightarrow$ $(0,0,0)$.

We give the proof of Theorem 2 in Section IV

For the case of complete security, i.e., $(\alpha, \beta, \gamma)=(0,0,0)$, Theorem 2 implies that Protocol 1 from CSSPIR is CSNSS. Furthermore, Protocol 1 converts a linear CSSPIR protocol into a linear CSNSS protocol as follows. 
Corollary 1. Let $\Phi_{\mathrm{SPIR}}^{\mathrm{m}}$ be a linear ( $\left.\mathfrak{A}, \mathfrak{B}, \mathrm{n}, \mathrm{f}\right)-C S S P I R$ protocol with rate $R_{\mathrm{PIR}}$, shared randomness rate $R_{U_{\mathrm{SPIR}} \text {, and }}$ position map $\tau$. Then, the NSS protocol $\hat{\Phi}_{\mathrm{NSS}}^{\mathrm{m}}\left[\Phi_{\mathrm{SPIR}}^{\mathrm{m}}\right]$ defined in Protocol 1 with $r^{*}=0, m_{[2: \mathrm{f}]}^{*}=0$ is a linear $(\mathfrak{A}, \mathfrak{B}, \mathrm{n})$ CSNSS protocol with SS rate $R_{\mathrm{SS}}=R_{\mathrm{PIR}}$, randomness rate $R_{U_{\mathrm{NSS}}}=R_{U_{\mathrm{SPIR}}}$, and position map $\tau$.

We give the proof of Corollary 1 in Section IV

In Corollary 1, the values of $r^{*}$ and $m_{[2: \mathrm{f}]}^{*}$ is chosen as zero instead of (17) and (18). This choice is justified by the following lemma.

Lemma 1. For any ( $\mathfrak{A}, \mathfrak{B}, \mathrm{n}, \mathrm{f})-C S S P I R$, any $k \in[\mathrm{f}]$, any $r \in \mathcal{R}$, and any $\mathcal{B} \in \mathfrak{B}$, we have

$$
I\left(M_{[\mathrm{n}]} ; D_{\mathcal{B}} \mid K=k, R=r\right)=0 .
$$

Proof. From the chain rule of the mutual information, we have

$$
\begin{aligned}
& I\left(M_{[\mathrm{n}]} ; D_{\mathcal{B}} \mid K=k, R=r\right) \\
& =I\left(M_{k} ; D_{\mathcal{B}} \mid K=k, R=r\right) \\
& \quad+I\left(M_{[\mathrm{n}] \backslash\{k\}} ; D_{\mathcal{B}} \mid M_{k}, K=k, R=r\right)
\end{aligned}
$$

The second term of (21) is zero from

$$
\begin{aligned}
& I\left(M_{[\mathrm{n}] \backslash\{k\}} ; D_{\mathcal{B}} \mid M_{k}, K=k, R=r\right) \\
& =I\left(M_{[\mathrm{n}] \backslash\{k\}} ; D_{\mathcal{B}} \mid K=k, R=r\right)=0,
\end{aligned}
$$

where the first equality follows from the correctness condition and the second equality is from the server secrecy of the CSSPIR protocol. The first term of (21) is zero as follows. From the user secrecy of the CSSPIR protocol, the answers $D_{\mathcal{B}}$ are generated independently of $K$, which implies that

$$
I\left(M_{k} ; D_{\mathcal{B}} \mid K=k, R=r\right)=I\left(M_{k} ; D_{\mathcal{B}} \mid K=k^{\prime}, R=r\right)=0,
$$

where the last equality follows from the server secrecy. Thus, we obtain the desired statement.

Next, we give the proof idea of Theorem 2 with Lemma 1 If we only consider the case of complete security, Theorem 2 for CSSPIR is simply proved as follows. First, we prove the complete correctness of the induced NSS protocol. For $\mathcal{A} \in \mathfrak{A}$, the first file $M_{1}$ is recovered from $D_{\mathcal{A}}$ since the answers $D_{\mathcal{A}}$ are generated from the SPIR protocol $\Phi_{\text {SPIR }}^{\mathrm{m}}$ for the case $K=1$, and the randomness $R=r^{*}$ is publicly known. Thus, the secret $L=M_{1}$ of the NSS protocol $\hat{\Phi}_{\mathrm{NSS}}^{\mathrm{m}}\left[\Phi_{\mathrm{SPIR}}^{\mathrm{m}}\right]$ is recovered from the shares $S_{\mathcal{A}}=D_{\mathcal{A}}$. Next, we prove the complete secrecy against forbidden sets $\mathcal{B} \in \mathfrak{B}$. From Lemma 1, we have $I\left(M_{1} ; D_{\mathcal{B}} \mid M_{[2: \mathrm{f}]}=m_{[2: \mathrm{f}]}^{*}, K=1, R=r^{*}\right)=0$, which implies that the shares $S_{\mathcal{B}}=D_{\mathcal{B}}$ have no information of the secret $L=M_{1}$. Thus, Theorem 2 for CSSPIR is proved.

Now, we discuss the achievable rate and capacity of CSSPIR. From Theorem 2 for $(\alpha, \beta, \gamma)=(0,0,0)$, we obtain the following corollary.

Corollary 2. If there is an $(\mathfrak{A}, \mathfrak{B}, \mathrm{n}, \mathrm{f})-C S S P I R$ protocol with PIR rate $R_{\mathrm{PIR}}$ and shared randomness rate $R_{U_{\mathrm{SPIR}}}$, the $S S$ rate $R_{\mathrm{PIR}}$ is achievable for $(\mathfrak{A}, \mathfrak{B}, \mathrm{n})-N S S$ with randomness rate $R_{U_{\mathrm{NSS}}}=R_{U_{\mathrm{SPIR}}}$.

An upper bound of SS rate for $(\mathfrak{A}, \mathfrak{B}, n)-C S N S S$ is proved in [12], [13], [14] as follows.
Proposition 1 ([12], [13], [14]). For any $\mathfrak{A}, \mathfrak{B} \subset 2^{[\mathrm{n}]}$, let $\delta(\mathfrak{A}, \mathfrak{B}):=\min \{|\mathcal{A}-\mathcal{B}| \mid \mathcal{A} \in \mathfrak{A}, \mathcal{B} \in \mathfrak{B}\}$. Any $(\mathfrak{A}, \mathfrak{B}, \mathrm{n})-$ CSNSS protocol $\Phi_{\mathrm{NSS}}^{\mathrm{m}}$ satisfies

$$
R_{\mathrm{SS}}\left(\Phi_{\mathrm{NSS}}^{\mathrm{m}}\right) \leq \frac{1}{\mathrm{n}} \delta(\mathfrak{A}, \mathfrak{B}) .
$$

In particular, for $(\mathrm{r}, \mathrm{t}, \mathrm{n})-C S N S S, R_{\mathrm{SS}} \leq \mathrm{r}-\mathrm{t}$.

As a corollary of Theorem 2 and Proposition 1 we obtain an upper bound of the CSSPIR capacity.

Corollary 3. For any $\mathfrak{A}, \mathfrak{B} \subset 2^{[\mathrm{n}]}$ and $\delta(\mathfrak{A}, \mathfrak{B})$ defined in Proposition 1$]$ we have

$$
C_{\mathrm{SPIR}}^{(\mathfrak{A}, \mathfrak{B}, \mathrm{n}, \mathrm{f})} \leq C_{\mathrm{NSS}}^{(\mathfrak{A}, \mathfrak{B}, \mathrm{n})} \leq \frac{1}{\mathrm{n}} \delta(\mathfrak{A}, \mathfrak{B})
$$

Corollary 3 is applicable for any access structure and is simply characterized by the access structure. For example, when a CSSPIR protocol has the access structure $\mathrm{n}=3$, $\mathfrak{A}=\{\{2,3\},\{1,2,3\}\}$, and $\mathfrak{B}=\{\emptyset,\{1\},\{2\},\{3\},\{1,2\}\}$ given in Example 1, Corollary 3 implies that the SPIR rate is upper bounded by $1 / 3$ because $n=3$ and $\delta(\mathfrak{A}, \mathfrak{B})=|\{2,3\}-\{2\}|=$ 1 .

Furthermore, the upper bound in Corollary 3 is tight for the threshold case as follows.

Corollary 4. The capacity of $\mathrm{n}$-server CSSPIR with $\mathrm{r}$ responsive servers and $\mathrm{t}$ colluding servers is $(\mathrm{r}-\mathrm{t}) / \mathrm{n}$.

The converse part is proved by Corollary 3 The achievability part of Corollary 4 follows from the linear CSSPIR protocol of Tajeddine et al. [28]. Tajeddine et al. [28] constructed a protocol of symmetric/non-symmetric CSSPIR from coded storage with colluding, byzantine, and unresponsive servers, with PIR rate

$$
\frac{r-k-2 b-t+1}{n}
$$

where $r$ is the number of responding servers, $k$ is the code rate of the coded storage, $b$ is the number of byzantine servers. When $(k, b)=(1,0)$, their protocol is an $(r, t, n, f)$-CSSPIR protocol and achieves the PIR rate $(r-t) / n$.

Holzbaur et al. [27, Theorem 4] proved that the rate 25] is optimal for linear CSSPIR with additive randomness. Thus, when $(k, b)=(1,0)$, the capacity for linear CSSPIR with additive randomness is the same as Corollary 4. Our result generalizes this result because Corollary 4 holds without the assumptions of the linearity of protocols and the additivity of the randomness.

Remark 5. The same implication from SPIR to NSS of Theorem 2 is applicable for multi-round SPIR by the same proof. Especially, since the multi-round SPIR capacity is greater than the one-round SPIR capacity, our result also implies that the multi-round capacity of $(r, t, n)$-CSSPIR is $(r-t) / n$ which is the same as the one-round capacity. Moreover, Theorem 2 is also applicable even for multi-round SPIR with coded database. However, for simplicity, this paper only discusses one-round protocols when all files replicated in each server. 


\section{B. Conversion from linear CSNSS to MMSP}

In this subsection, we give the conversion from linear CSNSS to MMSP.

Protocol 2. Let $\Phi_{\mathrm{NSS}}^{\mathrm{m}}$ be a linear CSNSS protocol defined from a linear encoder $f: \mathbb{F}_{q}^{\mathrm{x}+\mathrm{y}} \rightarrow \mathbb{F}_{q}^{\mathrm{z}}$ and a map $\tau:[\mathrm{z}] \rightarrow[\mathrm{n}]$. Let $\mathrm{G}_{f}$ be the matrix representation of the linear map $f$. Then, an MMSP $\hat{\mathcal{P}}\left[\Phi_{\mathrm{NSS}}^{\mathrm{m}}\right]:=\left(\mathrm{G}_{f}, \tau\right)$ is defined.

Theorem 3. Given a linear $(\mathfrak{A}, \mathfrak{B}, \mathrm{n})$-CSNSS protocol $\Phi_{\mathrm{NSS}}^{\mathrm{m}}$ with SS rate $R_{\mathrm{SS}}$, the MMSP $\hat{\mathcal{P}}\left[\Phi_{\mathrm{NSS}}^{\mathrm{m}}\right]$ defined in Protocol 2 is an $(\mathfrak{A}, \mathfrak{B}, \mathrm{n})-M M S P$ with $M M S P$ rate $R_{\mathrm{MMSP}}=R_{\mathrm{SS}}$.

We give the proof of Theorem 3 in Section $\square$

\section{Conversion from MMSP to projected linear CSSPIR}

In this subsection, we give the conversion from MMSP to projected linear CSSPIR. First, we define a projected linear SPIR protocol from MMSP.

Protocol 3. Let $\mathcal{P}=(\mathrm{G}, \tau)$ be an MMSP. We denote $\mathrm{G}=$ $\left(\mathrm{G}^{\prime}, \mathrm{G}^{\prime \prime}\right) \in \mathbb{F}_{q}^{\mathrm{zx}} \times \mathbb{F}_{q}^{\mathrm{z} \times \mathrm{y}}$. Let $\mathrm{f}$ be an arbitrary positive integer at least 2. Then, a projected linear CSSPIR protocol $\hat{\Phi}_{\mathrm{SPIR}}^{\mathrm{m}}[\mathcal{P}]$ is defined by the triplet $\left(\mathrm{G}^{\prime \prime}, \mathrm{G}^{\prime}, \tau\right)$ of randomness encoder $\mathrm{G}^{\prime \prime} \in \mathbb{F}_{q}^{\mathrm{z} \times \mathrm{y}}$, user's query encoder $\mathrm{G}^{\prime} \in \mathbb{F}_{q}^{\mathrm{z} \times \mathrm{x}}$, and position map $\tau:[\mathrm{z}] \rightarrow[\mathrm{n}]$.

The SPIR protocol defined in Protocol 3 is completely secure by the following theorem.

Theorem 4. Let $\mathcal{P}$ be an $(\mathfrak{A}, \mathfrak{B}, \mathrm{n})-M M S P$ with a matrix $\mathrm{G} \in$ $\mathbb{F}_{q}^{\mathrm{Z} \times(\mathrm{x}+\mathrm{y})}$, a position map $\tau:[\mathrm{z}] \rightarrow[\mathrm{x}]$, and the rate $R_{\mathrm{MMSP}}=$ $\mathrm{x} / \mathrm{z}$. Then, the SPIR protocol $\hat{\Phi}_{\mathrm{SPIR}}^{\mathrm{m}}[\mathcal{P}]$ defined in Protocol 3 is an $(\mathfrak{A}, \mathfrak{B}, \mathrm{n}, \mathrm{f})$-CSSPIR protocol $\Phi_{\mathrm{SPIR}}^{\mathrm{m}}$ for any $\mathrm{f} \geq 2$ with PIR rate $R_{\mathrm{PIR}}=R_{\mathrm{MMSP}}=\mathrm{x} / \mathrm{z}$ and shared randomness rate $\mathrm{y} / \mathrm{x}$.

We give the proof of Theorem 4 in Section VI

\section{Equivalence of linear CSSPIR, linear CSNSS, and MMSP}

Combining Corollary 1, Theorem 3, and Theorem 4, we obtain the equivalence of linear CSSPIR, linear CSNSS, and MMSP.

Corollary 5. Let $\mathrm{n}$ be a positive integer at least 2 , ( $\mathfrak{A}, \mathfrak{B})$ be an access structure on [n], and $\tau$ be a map from [z] to [n].

The following conditions are equivalent.

(a) For some $\mathrm{f} \geq 2$, there exists a linear $(\mathfrak{A}, \mathfrak{B}, \mathrm{n}, \mathrm{f})$-CSSPIR protocol with the position map $\tau$, the rate $R_{\mathrm{PIR}}=\mathrm{x} / \mathrm{z}$, and the shared randomness rate $R_{U \text { SPIR }}=\mathrm{y} / \mathrm{x}$.

(b) There exists a linear $(\mathfrak{A}, \mathfrak{B}, \mathrm{n})-C S N S S$ protocol with the position map $\tau$, the rate $R_{\mathrm{SS}}=\mathrm{x} / \mathrm{z}$, and the randomness rate $R_{U_{\mathrm{NSS}}}=\mathrm{y} / \mathrm{x}$.

(c) There exists a $(\mathfrak{A}, \mathfrak{B}, \mathrm{n})$-MMSP with the matrix $\mathrm{G} \in$ $\mathbb{F}_{q}^{\mathrm{Zx}(\mathrm{x}+\mathrm{y})}$ the position map $\tau$, and rate $R_{\mathrm{MMSP}}=\mathrm{x} / \mathrm{z}$.

(d) For any $f \geq 2$, there exists a projected linear $(\mathfrak{A}, \mathfrak{B}, \mathrm{n}, \mathrm{f})$ CSSPIR protocol with the position map $\tau$, the rate $R_{\mathrm{PIR}}=$ $\mathrm{x} / \mathrm{z}$, and the shared randomness rate $R_{U_{\mathrm{SPIR}}}=\mathrm{y} / \mathrm{x}$.

Corollary 5 is proved as follows. The relations $(a) \Rightarrow(b)$, (b) $\Rightarrow(c)$, and $(c) \Rightarrow(d)$ follow from Corollary 1
Theorem [3, and Theorem 4, respectively, and $(d) \Rightarrow(a)$ is trivial.

For any linear CSNSS protocol $\Phi_{\mathrm{NSS}}^{\mathrm{m}}$, we have

$$
\hat{\Phi}_{\mathrm{NSS}}^{\mathrm{m}}\left[\hat{\Phi}_{\mathrm{SPIR}}^{\mathrm{m}}\left[\hat{\mathcal{P}}\left[\Phi_{\mathrm{NSS}}^{\mathrm{m}}\right]\right]\right]=\Phi_{\mathrm{NSS}}^{\mathrm{m}} .
$$

That is, the composite map $\hat{\Phi}_{\mathrm{NSS}}^{\mathrm{m}} \circ \hat{\Phi}_{\mathrm{SPIR}}^{\mathrm{m}} \circ \hat{\mathcal{P}}$ is the identity map on linear NSS. Similarly, the composite map $\hat{\Phi}_{\mathrm{SPIR}}^{\mathrm{m}} \circ \hat{\mathcal{P}} \circ$ $\hat{\Phi}_{\mathrm{NSS}}^{\mathrm{m}}$ is the projection $\hat{p}$ into projected linear SPIR, defined in Definition 9 i.e., for any a projected linear CSSPIR protocol $\Phi_{\text {SPIR }}^{\mathrm{m}}$, we have

$$
\hat{\Phi}_{\mathrm{SPIR}}^{\mathrm{m}}\left[\hat{\mathcal{P}}\left[\hat{\Phi}_{\mathrm{NSS}}^{\mathrm{m}}\left[\Phi_{\mathrm{SPIR}}^{\mathrm{m}}\right]\right]\right]=\hat{p}\left(\Phi_{\mathrm{SPIR}}^{\mathrm{m}}\right)=\Phi_{\mathrm{SPIR}}^{\mathrm{m}} .
$$

A linear CSSPIR protocol $\Phi_{\text {SPIR }}^{\mathrm{m}}$ and its projected protocol $\hat{p}\left(\Phi_{\mathrm{SPIR}}^{\mathrm{m}}\right)$ are compared as follows. In the two protocols, the sizes of queries, answers, and random seed are the same. However, from definitions of linear SPIR and projected linear SPIR, the projected protocol is more concisely described. That is, whereas the random query matrices $Q \in \mathbb{F}_{q}^{\mathrm{zxfx}}$ are necessary to be characterized in the original CSSPIR protocol $\Phi_{\mathrm{SPIR}}^{\mathrm{m}}$, all queries of the projected protocol $\hat{p}\left(\Phi_{\text {SPIR }}^{\mathrm{m}}\right)$ can be described only with a small matrix $J \in \mathbb{F}_{q}^{z \times x}$. On the other hand, since the size of user's private randomness $R \in \mathbb{F}_{q}^{y \times f x}$ in the projected protocol is increasing with the number of the files $f$, one possible advantage of linear CSSPIR protocols would be the smaller size of this randomness.

Next, we discuss the relation between projected linear CSSPIR and linear CSNSS as follows. In both protocols, the minimum information to describe encoders is two matrices in $\mathbb{F}_{q}^{\mathrm{ZXX}}$ and $\mathbb{F}_{q}^{\mathrm{Zxy}}$. The matrix in $\mathbb{F}_{q}^{\mathrm{zx}}$ is used for encoding messages (targeted file for CSSPIR and the secret for CSNSS) and the matrix in $\mathbb{F}_{q}^{\mathrm{Z} \times \mathrm{y}}$ for encoding randomness (and non-targeted files for CSSPIR). From this similarity, the server secrecy of CSSPIR and the secrecy of CSNSS are guaranteed in the same context. On the other hand, the uniform randomness for CSNSS is the dealer's randomness $U_{\text {NSS }} \in \mathbb{F}_{q}^{y}$, while that for CSSPIR is the random seed $U_{\text {SPIR }} \in \mathbb{F}_{q}^{y}$ and the user's private randomness $R \in \mathbb{F}_{q}^{y \times f x}$. In CSSPIR, the additional randomness $R$ is required for guaranteeing the user secrecy.

Farràs et al. proved the existence of a linear CSNSS protocol for any access structure [16].

Proposition 2 ([16]). For any access structure (A), $\mathfrak{B})$, there exists a linear $(\mathfrak{A}, \mathfrak{B}, \mathrm{n})$-CSNSS protocol.

Combining Proposition 2 and Corollary [5, we obtain the following corollary.

Corollary 6. For any access structure $(\mathfrak{A}, \mathfrak{B})$ on $[\mathrm{n}]$ and any $\mathrm{f} \geq 2$, there exists an $(\mathfrak{A}, \mathfrak{B}, \mathrm{n}, \mathrm{f})$-CSSPIR protocol.

\section{PROOF OF CONVERSION FROM SPIR TO NSS}

In this section, we prove Theorem 2 and Corollary 2

\section{A. Proof of Theorem 2}

For the proof, we prepare the following lemma, which extends Lemma 1 to the incomplete secrecy case. 
Lemma 2. For any $(\mathfrak{A}, \mathfrak{B}, \mathrm{n})-S P I R$ protocol $\Phi_{\mathrm{SPIR}}^{\mathrm{m}}$, any $\mathcal{B} \in$ $\mathfrak{B}$, and $r^{*}$ defined in (17), we have

$I\left(M_{1} ; D_{\mathcal{B}} \mid M_{[2: f]}, R=r^{*}, K=1\right)$

$\leq 2 \beta+(1-\alpha+4 \sqrt{2 \gamma \mathrm{f}}) \log \mathrm{m}+2 h_{2}(\sqrt{2 \gamma \mathrm{f}})+h_{2}(1-\alpha)+\log \alpha$.

The proof of Lemma 2 is given in Appendix B Now, we prove Theorem 2 .

Proof of Theorem 2 The correctness of the NSS protocol $\hat{\Phi}_{\mathrm{NSS}}^{\mathrm{m}}\left[\Phi_{\mathrm{SPIR}}^{\mathrm{m}}\right]$ is upper bounded by $\alpha$ because we have

$$
\begin{aligned}
\alpha\left(\hat{\Phi}_{\mathrm{NSS}}^{\mathrm{m}}\left[\Phi_{\mathrm{SPIR}}^{\mathrm{m}}\right]\right) \stackrel{(a)}{=} \max _{\mathcal{A} \in \mathscr{A}} \operatorname{Pr}_{M_{1}, D_{\mathcal{A}}}\left[M_{1} \neq \hat{m}_{D_{\mathcal{A}}, r^{*}, k=1}^{\mathrm{ML}}\right] \\
\\
\stackrel{(b)}{\leq} \alpha\left(\Phi_{\mathrm{SPIR}}^{\mathrm{m}}\right),
\end{aligned}
$$

where $(a)$ follows from the definition of Protocol 1 and $(b)$ follows the correctness condition of the SPIR protocol $\Phi_{\mathrm{SPIR}}^{\mathrm{m}}$. The secrecy $\beta\left(\hat{\Phi}_{\mathrm{NSS}}^{\mathrm{m}}\left[\Phi_{\mathrm{SPIR}}^{\mathrm{m}}\right]\right)$ is upper bounded as

$I\left(M_{1} ; D_{\mathcal{B}} \mid M_{[2: f]}=m_{[2: \mathrm{f}]}^{*}, R=r^{*}, K=1\right)$

(a)

$\stackrel{\text { (a) }}{\leq} I\left(M_{1} ; D_{\mathcal{B}} \mid M_{[2: f]}, R=r^{*}, K=1\right)$

$\stackrel{(b)}{\leq} 2 \beta+(1-\alpha+4 \sqrt{2 \gamma \mathrm{f}}) \log \mathrm{m}+2 h_{2}(\sqrt{2 \gamma \mathrm{f}})+h_{2}(1-\alpha)+\log \alpha$

$=\xi(\alpha, \beta, \gamma)$,

where $(a)$ follows from the choice of $m_{[2: f]}^{*}$ in (18) and $(b)$ follows from Lemman Thus, the protocol $\hat{\Phi}_{\mathrm{NSS}}^{\mathrm{m}}\left[\Phi_{\mathrm{SPIR}}^{\mathrm{m}}\right]$ is an $(\mathfrak{A}, \mathfrak{B}, \mathrm{n}, \mathrm{f})$-NSS protocol with the desired security parameters $(\alpha, \xi(\alpha, \beta, \gamma))$. The SS rate of Protocol 11 is

$$
R_{\mathrm{SS}}=\frac{\mathrm{n} \log \mathrm{m}}{\sum_{j \in[\mathrm{n}]} \log \left|\mathcal{S}_{j}\right|}=\frac{\mathrm{n} \log \mathrm{m}}{\sum_{j \in[\mathrm{n}]} \log \left|\mathcal{D}_{j}\right|}=R_{\mathrm{PIR}},
$$

which proves Theorem 2 .

\section{B. Proof of Theorem 2}

The proof of Corollary 1 is as follows. The conditions for the access structure, security, and rate follow from Theorem 2 Thus, it is enough to prove that the NSS protocol $\hat{\Phi}_{\mathrm{NSS}}^{\mathrm{m}}\left[\Phi_{\mathrm{SPIR}}^{\mathrm{m}}\right]$ is linear. To prove the linearity, we first analyze the simulation of the linear SPIR protocol $\Phi_{\text {SPIR }}^{m}$ in Protocol 1 , and then, we prove that the NSS protocol $\Phi_{\mathrm{NSS}}^{\mathrm{m}}\left[\Phi_{\mathrm{SPIR}}^{\mathrm{m}}\right]$ is linear.

First, we analyze Protocol 11 with parameters defined in Corollary 1. In Corollary 1, the linear CSSPIR protocol $\Phi_{\text {SPIR }}^{\mathrm{m}}$ is simulated with $K=1, R=0$, and $M_{[2: f]}=0$. Since the query is determined by $K$ and $R$, the simulated query is fixed as a matrix $Q \in \mathbb{F}_{q}^{\mathrm{zxfx}}$. We denote $\mathrm{Q}=\left(\mathrm{Q}^{\prime}, \mathrm{Q}^{\prime \prime}\right) \in \mathbb{F}_{q}^{\mathrm{zxx}} \times \mathbb{F}_{q}^{\mathrm{Zx}(\mathrm{f}-1) \mathrm{x}}$. With the uniform randomness $U_{\text {SPIR }} \in \mathbb{F}_{q}^{y}$ and the randomness encoder $\mathrm{H} \in \mathbb{F}_{q}^{\mathrm{z} \times \mathrm{y}}$ defined in Definition[7, the answers of $\Phi_{\mathrm{SPIR}}^{\mathrm{m}}$ are written as

$$
\left(\begin{array}{c}
D_{1} \\
\vdots \\
D_{\mathrm{n}}
\end{array}\right)=\mathrm{Q} M+T \stackrel{(a)}{=} \mathrm{Q}^{\prime} M_{1}+\mathrm{H} U_{\mathrm{SPIR}}=\left(\mathrm{Q}^{\prime}, \mathrm{H}\right)\left(\begin{array}{c}
M_{1} \\
U_{\mathrm{SPIR}}
\end{array}\right) \in \mathbb{F}_{q}^{\mathrm{z}}
$$

$$
D_{j}=\left(\mathrm{Q}^{\prime}, \mathrm{H}\right)_{\tau^{-1}(j)}\left(\begin{array}{c}
M_{1} \\
U_{\text {SPIR }}
\end{array}\right) \in \mathbb{F}_{q}^{\left|\tau^{-1}(j)\right|},
$$

where $(a)$ follows from the condition $M_{2}=\cdots=M_{\mathrm{f}}=0$ of Corollary 1

Next, we prove that the NSS protocol $\hat{\Phi}_{\mathrm{NSS}}^{\mathrm{m}}\left[\Phi_{\mathrm{SPIR}}^{\mathrm{m}}\right]$ is linear. The shares of $\hat{\Phi}_{\mathrm{NSS}}^{\mathrm{m}}\left[\Phi_{\mathrm{SPIR}}^{\mathrm{m}}\right]$ are generated as $S_{j}=D_{j}$ in (38) while the secret $L$ of NSS is embedded as $M_{1}=L \in \mathbb{F}_{q}^{\mathrm{X}}$. Thus, $\hat{\Phi}_{\mathrm{NSS}}^{\mathrm{m}}\left[\Phi_{\mathrm{SPIR}}^{\mathrm{m}}\right]$ corresponds to the linear NSS protocol with the dealer's private randomness $U_{\mathrm{NSS}}=U_{\mathrm{SPIR}} \in \mathbb{F}_{q}^{\mathrm{y}}$, linear encoder $\left(\mathrm{Q}^{\prime}, \mathrm{H}\right) \in \mathbb{F}_{q}^{\mathrm{zx}(\mathrm{x}+\mathrm{y})}$, and the same position map $\tau$ as $\Phi_{\mathrm{SPIR}}^{\mathrm{m}}$.

\section{Proof of CONVERSion From LINEAR CSNSS to MMSP}

In this section, we prove Theorem 3, i.e., the MMSP $\hat{\mathcal{P}}\left[\Phi_{\mathrm{NSS}}^{\mathrm{m}}\right]$ defined in Protocol 2 from a linear $(\mathfrak{A}, \mathfrak{B}, \mathrm{n})$-CSNSS protocol is an $(\mathfrak{A}, \mathfrak{B}, n)$-MMSP. Before the proof, we prepare the following proposition and lemma.

Proposition 3. For any random variable $X \in \mathbb{F}_{q}^{n}$ and $\mathrm{A} \in$ $\mathbb{F}_{q}^{m \times n}$, we have

$$
H(\mathrm{AX}) \leq \operatorname{rank} \mathrm{A} \log q .
$$

When the distribution of $X$ is uniform, the equivalence of (39) holds.

Lemma 3. The rejection condition of an MMSP $\mathcal{P}$ is equivalent to

$$
\operatorname{rank} \mathrm{G}_{\tau^{-1}(\mathcal{B})}^{\prime \prime}=\operatorname{rank} \mathrm{G}_{\tau^{-1}(\mathcal{B})} \quad \forall \mathcal{B} \in \mathfrak{B} .
$$

Proof. First, from definition, the rejection condition of the MMSP $\mathcal{P}$ is equivalent to

$$
\mathrm{x}+\operatorname{rank} \mathrm{G}_{\tau^{-1}(\mathcal{B})}=\operatorname{rank}\left(\begin{array}{cc}
\mathrm{I}_{\mathrm{x}} & \mathrm{O}_{\mathrm{x} \times \mathrm{y}} \\
\mathrm{G}_{\tau^{-1}(\mathcal{B})}
\end{array}\right) \quad \forall \mathcal{B} \in \mathfrak{B} .
$$

Thus, we prove the equivalence between (40) and (41).

The direction from (40) to (41) is proved as

$$
\begin{aligned}
& \mathrm{X}+\operatorname{rank} \mathrm{G}_{\tau^{-1}(\mathcal{B})}=\mathrm{X}+\operatorname{rank} \mathrm{G}_{\tau^{-1}(\mathcal{B})}^{\prime \prime} \\
& =\operatorname{rank}\left(\begin{array}{cc}
\mathrm{I}_{\mathrm{X}} & \mathrm{O}_{\mathrm{x} \times \mathrm{y}} \\
\mathrm{O}_{\mathrm{z} \times \mathrm{x}} & \mathrm{G}_{\tau^{-1}(\mathcal{B})}^{\prime \prime}
\end{array}\right)=\operatorname{rank}\left(\begin{array}{cc}
\mathrm{I}_{\mathrm{X}} & \mathrm{O}_{\mathrm{x} \times \mathrm{y}} \\
G_{\tau^{-1}(\mathcal{B})}
\end{array}\right)
\end{aligned}
$$

which implies (41). The direction from (41) to (40) is proved as

$$
\begin{aligned}
& x+\operatorname{rank} G_{\tau^{-1}(\mathcal{B})} \stackrel{(a)}{=} \operatorname{rank}\left(\begin{array}{cc}
\mathrm{I}_{\mathrm{x}} & \mathrm{O}_{\mathrm{x} \times \mathrm{y}} \\
\mathrm{G}_{\tau^{-1}(\mathcal{B})}
\end{array}\right) \\
& =\operatorname{rank}\left(\begin{array}{cc}
\mathrm{I}_{\mathrm{x}} & \mathrm{O}_{\mathrm{x} \times \mathrm{y}} \\
\mathrm{O}_{\mathrm{z} \times \mathrm{x}} & \mathrm{G}_{\tau^{-1}(\mathcal{B})}^{\prime \prime}
\end{array}\right)=\mathrm{x}+\operatorname{rank} \mathrm{G}_{\tau^{-1}(\mathcal{B})}^{\prime \prime},
\end{aligned}
$$

where (a) follows from the rejection condition. Thus, we obtain the desired statement.

Now, we prove Theorem 3 .

Proof of Theorem 3 The rates of $\hat{\mathcal{P}}\left[\Phi_{\mathrm{NSS}}^{\mathrm{m}}\right]$ and $\Phi_{\mathrm{NSS}}^{\mathrm{m}}$ are trivially $\mathrm{x} / \mathrm{z}$. In the following, we separately prove that $\hat{\mathcal{P}}\left[\Phi_{\mathrm{NSS}}^{\mathrm{m}}\right]$ accepts $\mathfrak{A}$ and rejects $\mathfrak{B}$.

First, we prove that the MMSP $\hat{\mathcal{P}}\left[\Phi_{\mathrm{NSS}}^{\mathrm{m}}\right]$ accepts $\mathfrak{A}$. Let $\mathcal{A} \in \mathfrak{A}$. Then, the completely secure correctness condition of the linear CSNSS guarantees that there exists a function $h$ such that $h\left(S_{\mathcal{A}}\right)=L$, i,e.,

$$
h\left(S_{\mathcal{A}}\right)=h\left(\mathrm{G}_{\tau^{-1}(\mathcal{A})}\left(\begin{array}{l}
L \\
R
\end{array}\right)\right)=L .
$$


Since (46) holds for any $L \in \mathbb{F}_{q}^{\mathrm{X}}$ and $R \in \mathbb{F}_{q}^{\mathrm{y}}, h$ is written as a linear map from $\operatorname{Im~}_{\tau^{-1}(\mathcal{A})}$ to $\mathbb{F}_{q}^{\mathrm{X}}$ and the associated matrix $\mathrm{K}_{h} \in \mathbb{F}_{q}^{\mathrm{x} \times\left|\tau^{-1}(\mathcal{A})\right|}$ of the function $h$ satisfies

$$
\mathrm{K}_{h} \mathrm{G}_{\tau^{-1}(\mathcal{A})}=\left(\mathrm{I}_{\mathrm{x}}, \mathrm{O}_{\mathrm{x} \times \mathrm{y}}\right) \text {, }
$$

where $I_{x}$ is the $x \times x$ identity matrix and $O_{x x y}$ is the $x \times y$ zero matrix. Thus, the row space of $\mathrm{G}_{\tau^{-1}(\mathcal{A})}$ includes the row space of $\left(\mathrm{I}_{\mathrm{x}}, \mathrm{O}_{\mathrm{x} \times \mathrm{y}}\right)$, which implies that the MMSP $\hat{\mathcal{P}}\left[\Phi_{\mathrm{NSS}}^{\mathrm{m}}\right]$ accepts $\mathfrak{A}$.

Next, we prove that the MMSP $\hat{\mathcal{P}}\left[\Phi_{\text {NSS }}^{\mathrm{m}}\right]$ rejects $\mathfrak{B}$. We denote $\mathrm{G}_{f}=\left(\mathrm{G}_{f}^{\prime}, \mathrm{G}_{f}^{\prime \prime}\right) \in \mathbb{F}_{q}^{\mathrm{zx}} \times \mathbb{F}_{q}^{\mathrm{z} \times \mathrm{y}}$. From Lemma 3 , the fact that $\hat{\mathcal{P}}\left[\Phi_{\mathrm{NSS}}^{\mathrm{m}}\right]$ rejects $\mathfrak{B}$ is equivalent to

$$
\operatorname{rank} \mathrm{G}_{f, \tau^{-1}(\mathcal{B})}^{\prime \prime}=\operatorname{rank} \mathrm{G}_{f, \tau^{-1}(\mathcal{B})} \quad \forall \mathcal{B} \in \mathfrak{B} .
$$

Thus, we prove (48) as follows: For any $\mathcal{B} \in \mathfrak{B}$, the MMSP $\hat{\mathcal{P}}\left[\Phi_{\mathrm{NSS}}^{\mathrm{m}}\right]$ satisfies

$$
\begin{aligned}
& \operatorname{rank} \mathrm{G}_{f, \tau^{-1}(\mathcal{B})} \log q \stackrel{(a)}{=} H\left(S_{\mathcal{B}}\right) \stackrel{(b)}{=} H\left(S_{\mathcal{B}} \mid L=\boldsymbol{\ell}\right) \\
& =H\left(\mathrm{G}_{f, \tau^{-1}(\mathcal{B})}^{\prime} L+\mathrm{G}_{\tau^{-1}(\mathcal{B})}^{\prime \prime} R \mid L=\boldsymbol{\ell}\right) \\
& =H\left(\mathrm{G}_{f, \tau^{-1}(\mathcal{B})}^{\prime \prime} R\right) \stackrel{(c)}{=} \operatorname{rank} \mathrm{G}_{f, \tau^{-1}(\mathcal{B})}^{\prime \prime} \log q
\end{aligned}
$$

where $(a)$ and $(c)$ follow from Proposition 3 and the uniform randomness of $L$ and $R$, and $(b)$ follows from the secrecy condition $I\left(L ; S_{\mathcal{B}}\right)=0$ of the CSNSS protocol. Therefore, the MMSP $\hat{\mathcal{P}}\left[\Phi_{\mathrm{NSS}}^{\mathrm{m}}\right]$ rejects $\mathfrak{B}$.

\section{PROOF OF CONVERSION FROM MMSP TO PROJECTED LINEAR CSSPIR}

In this section, the SPIR protocol $\hat{\Phi}_{\mathrm{SPIR}}^{\mathrm{m}}[\mathcal{P}]$ defined in Protocol 3 from an $(\mathfrak{A}, \mathfrak{B}, \mathrm{n})$-MMSP $\mathcal{P}$ is an $(\mathfrak{A}, \mathfrak{B}, \mathrm{n}, \mathfrak{f})$ CSSPIR protocol.

For the proof, we prepare the following lemma.

Lemma 4. Let $\mathcal{P}=(\mathrm{G}, \tau)$ be an $(\mathfrak{A}, \mathfrak{B}, \mathrm{n})-M M S P, X \in \mathbb{F}_{q}^{\mathrm{X}}$ be a random vector, and $Y \in \mathbb{F}_{q}^{y}$ be a uniform random vector. For $X \in[\mathrm{n}]$, we define

$$
Z_{\tau^{-1}(X)}=\mathrm{G}_{\tau^{-1}(\mathcal{X})}\left(\begin{array}{l}
X \\
Y
\end{array}\right) .
$$

Then, the relation

$$
H\left(Z_{\tau^{-1}(\mathcal{B})}\right)=H\left(Z_{\tau^{-1}(\mathcal{B})} \mid X=\mathbf{x}\right)
$$

holds for any $\mathcal{B} \in \mathfrak{B}$ and $\mathbf{x} \in \mathbb{F}_{q}^{\mathrm{X}}$, i.e., $I\left(Z_{\tau^{-1}(\mathcal{B})} ; X\right)=0$.

Proof. From Lemma 3 , the fact that $\mathcal{P}$ rejects $\mathfrak{B}$ is equivalent to

$$
\operatorname{rank} \mathrm{G}_{\tau^{-1}(\mathcal{B})}^{\prime \prime}=\operatorname{rank} \mathrm{G}_{\tau^{-1}(\mathcal{B})} \quad \forall \mathcal{B} \in \mathfrak{B} .
$$

From this relation, we obtain

$$
\begin{aligned}
& H\left(Z_{\tau^{-1}(\mathcal{B})} \mid X=\mathbf{x}\right)=H\left(\mathrm{G}_{\tau^{-1}(\mathcal{B})}^{\prime} \mathbf{x}+\mathrm{G}_{\tau^{-1}(\mathcal{B})}^{\prime \prime} Y\right) \\
& =H\left(\mathrm{G}_{\tau^{-1}(\mathcal{B})}^{\prime \prime} Y\right) \stackrel{(a)}{=} \operatorname{rank} \mathrm{G}_{\tau^{-1}(\mathcal{B})}^{\prime \prime} \log q \\
& \stackrel{(b)}{=} \operatorname{rank} \mathrm{G}_{\tau^{-1}(\mathcal{B})} \log q \stackrel{(c)}{=} H\left(Z_{\tau^{-1}(\mathcal{B})}\right),
\end{aligned}
$$

where $(a)$ and $(c)$ follow from Proposition 3 and $(b)$ follows from (54).
Now, we prove Theorem 4

Proof of Theorem 4. From the definition of Protocol 3, it is clear that the SPIR protocol $\hat{\Phi}_{\text {SPIR }}^{m}[\mathcal{P}]$ is projected linear and the PIR rate is $R_{\mathrm{PIR}}=\mathrm{x} / \mathrm{z}=R_{\mathrm{MMSP}}$. Thus, in the following, we separately prove that the SPIR protocol $\hat{\Phi}_{\mathrm{SPIR}}^{\mathrm{m}}[\mathcal{P}]$ has completely secure user secrecy, server secrecy, and correctness.

First, the user secrecy of $\hat{\Phi}_{\mathrm{SPIR}}^{\mathrm{m}}[\mathcal{P}]$ is proved as follows. The queries indexed by $\mathcal{B} \in \mathfrak{B}$ are written as

$$
Q_{\tau^{-1}(\mathcal{B})}=\mathrm{G}_{\tau^{-1}(\mathcal{B})}\left(\begin{array}{c}
\mathrm{E}_{k} \\
R
\end{array}\right) .
$$

From Lemma 4 and the uniform randomness of $R$, the distribution of $Q_{\tau^{-1}(\mathcal{B})}$ does not depend on the value of $k$, which implies the completely secure user secrecy.

Server secrecy of $\hat{\Phi}_{\text {SPIR }}^{m}[\mathcal{P}]$ is proved as follows. The answers indexed by $\mathcal{X} \subset[\mathrm{n}]$ are written as

$$
\begin{aligned}
D_{\mathcal{X}} & =Q_{\tau^{-1}(\mathcal{X})} M+\mathrm{G}_{\tau^{-1}(\mathcal{X})}^{\prime \prime} U_{\mathrm{SPIR}} \\
& =\mathrm{G}_{\tau^{-1}(\mathcal{X})}^{\prime} \mathrm{E}_{k} M+\mathrm{G}_{\tau^{-1}(\mathcal{X})}^{\prime \prime}\left(R M+U_{\mathrm{SPIR}}\right) \\
& =\mathrm{G}_{\tau^{-1}(\mathcal{X})}^{\prime} M_{k}+\mathrm{G}_{\tau^{-1}(\mathcal{X})}^{\prime \prime} U_{\mathrm{SPIR}}^{\prime} \\
& =\mathrm{G}_{\tau^{-1}(\mathcal{X})}\left(\begin{array}{c}
M_{k} \\
U_{\mathrm{SPIR}}^{\prime}
\end{array}\right) \in \mathbb{F}_{q}^{|X|},
\end{aligned}
$$

where $U_{\text {SPIR }}^{\prime}:=R M+U_{\text {SPIR }} \in \mathbb{F}_{q}^{\mathrm{y}}$. Since $U_{\text {SPIR }}$ is uniform randomness, $U_{\text {SPIR }}^{\prime}$ in 62 is also uniformly random. Thus, for any $\mathcal{X} \in[\mathrm{n}]$, the user's received information (62) does not depend on the files except for $M_{k}$, which implies the completely secure server secrecy.

Next, we prove the correctness of the SPIR protocol $\hat{\Phi}_{\text {SPIR }}^{\mathrm{m}}[\mathcal{P}]$. Since $\mathcal{P}$ accepts $\mathfrak{A}$, i.e., the row space of $\mathrm{G}_{\tau^{-1}(\mathcal{A})}$ contains $\mathbf{e}_{1}, \ldots, \mathbf{e}_{\mathrm{x}} \in \mathbb{F}_{q}^{\mathrm{x}+\mathrm{y}}$ for any $\mathcal{A} \in \mathfrak{A}$, there exists a matrix $\mathrm{K}[\mathcal{A}]$ such that

$$
\mathrm{K}[\mathcal{A}] \mathrm{G}_{\tau^{-1}(\mathcal{A})}=\left(\mathrm{I}_{\mathrm{x}}, \mathrm{O}_{\mathrm{x} \times \mathrm{y}}\right),
$$

where $I_{x}$ is the $x \times x$ identity matrix and $O_{x \times y}$ is the $x \times y$ zero matrix. Since (62) implies

$$
D_{\mathcal{A}}=\mathrm{G}_{\tau^{-1}(\mathcal{A})}\left(\begin{array}{c}
M_{k} \\
U_{\mathrm{SPIR}}^{\prime}
\end{array}\right),
$$

by applying $\mathrm{K}[\mathcal{A}]$ to the answers $\mathcal{D}_{\mathcal{A}}$, the user obtains $\mathrm{K}[\mathcal{A}] \mathcal{D}_{\mathcal{A}}=M_{k}$ correctly.

\section{EXAMPLES OF CONSTRUCTIONS}

\section{A. A construction with access structure in Example 1}

In this subsection, we give a simple example of a MMSP with the general access structure in Example 1.

The access structure in Example 1 is defined as $\mathrm{n}=3$, $\mathfrak{U}=\{\{2,3\},\{1,2,3\}\}$, and $\mathfrak{B}=\{\emptyset,\{1\},\{2\},\{3\},\{1,2\}\}$. We fix $x=1, y=2$, and $z=4$, and define an ( $\mathfrak{A}, \mathfrak{B}, n)$-MMSP by

$$
\begin{aligned}
\mathrm{G} & =\left(\begin{array}{lll}
0 & 1 & 2 \\
1 & 1 & 1 \\
0 & 1 & 1 \\
1 & 1 & 0
\end{array}\right) \in \mathbb{F}_{3}^{4 \times 3}, \\
\tau & =\{1 \mapsto 1,2 \mapsto 2,3 \mapsto 3,4 \mapsto 3\} .
\end{aligned}
$$


Then, we can confirm that this MMSP $\mathcal{P}=(\mathrm{G}, \tau)$ accepts $\mathfrak{A}$ and rejects $\mathfrak{B}$ as follows. To confirm that $\mathcal{P}$ accepts $\mathfrak{A}$, it is enough to confirm if

$$
\mathcal{E}=\{(1,0,0)\} \subset \operatorname{rowspan} \mathrm{G}_{\mathcal{A}}
$$

for $\mathcal{A}=\{2,3\}$. Since

$$
\mathrm{G}_{\tau^{-1}(\{2,3\})}=\mathrm{G}_{\{2,3,4\}}=\left(\begin{array}{lll}
1 & 1 & 1 \\
0 & 1 & 1 \\
1 & 1 & 0
\end{array}\right)
$$

satisfies the above inclusion, $\mathcal{P}$ accepts $\mathfrak{A}$. To confirm that $\mathcal{P}$ accepts $\mathfrak{B}$, we need to confirm

$$
\operatorname{span} \mathcal{E} \cap \operatorname{rowspan} \mathrm{G}_{\tau^{-1}(\mathcal{B})}=\{0\}
$$

for $\mathcal{B}=\{1,2\}$ and $\mathcal{B}=\{3\}$. Since both

$$
\begin{aligned}
G_{\tau^{-1}(\{1,2\})} & =G_{\{1,2\}}=\left(\begin{array}{lll}
0 & 1 & 2 \\
1 & 1 & 1
\end{array}\right), \\
G_{\tau^{-1}(\{3\})} & =G_{\{3,4\}}=\left(\begin{array}{lll}
0 & 1 & 1 \\
1 & 1 & 0
\end{array}\right),
\end{aligned}
$$

satisfy the above property, $\mathcal{P}$ rejects $\mathfrak{B}$. Therefore, $\mathcal{P}$ is an $(\mathfrak{A}, \mathfrak{B}, \mathrm{n})$-MMSP. The rate of $\mathcal{P}$ is $R_{\mathrm{MMSP}}=\mathrm{x} / \mathrm{z}=1 / 4$.

From this MMSP $\mathcal{P}$ and Protocols 1, 2, and 3, we can also obtain $(\mathfrak{A}, \mathfrak{B}, n, f)$-SPIR and $(\mathfrak{A}, \mathfrak{B}, n)$-NSS protocols for the access structure in Example 1 and any $f \geq 2$. The induced SPIR and NSS protocols has the same rate $x / z=1 / 4$ and randomness rate $\mathrm{y} / \mathrm{x}=2$.

\section{B. Optimal construction of $(\mathrm{r}, \mathrm{t}, \mathrm{n})-\operatorname{CSNSS}$ and $(\mathrm{r}, \mathrm{t}, \mathrm{n}, \mathrm{f})$ - CSSPIR}

In this subsection, we construct $(r, t, n)$-CSNSS and $(r, t, n, f)$-CSSPIR protocols with optimal rates from a $(r, t, n)-$ MMSP. The proposed protocols are already proposed by Yamamoto [11] and by Tajeddine et al. [28] (as a special case), but we construct these protocols from MMSPs.

In the following, we assume that the size of the finite field $\mathbb{F}_{q}$ is at least $\mathrm{n}$. We define an MMSP $\mathcal{P}=(\mathrm{G}, \tau)$ by the Vandermonde matrix, which is also the generator matrix of a Reed-Solomon code [59]. Let $(x, y, z)=(r-t, t, n)$ and $\tau=\operatorname{id}_{[n]}$. Define

$$
\mathrm{G}=\left(\begin{array}{cccc}
1 & \alpha_{1} & \cdots & \alpha_{\mathrm{x}+\mathrm{y}-1} \\
1 & \alpha_{1}^{2} & \cdots & \alpha_{\mathrm{x}+\mathrm{y}-1}^{2} \\
\vdots & \vdots & \ddots & \vdots \\
1 & \alpha_{1}^{z} & \cdots & \alpha_{\mathrm{x}+\mathrm{y}-1}^{\mathrm{z}}
\end{array}\right)=\left(\begin{array}{cccc}
1 & \alpha_{1} & \cdots & \alpha_{\mathrm{r}-1} \\
1 & \alpha_{1}^{2} & \cdots & \alpha_{\mathrm{r}-1}^{2} \\
\vdots & \vdots & \ddots & \vdots \\
1 & \alpha_{1}^{\mathrm{n}} & \cdots & \alpha_{\mathrm{r}-1}^{\mathrm{n}}
\end{array}\right) .
$$

Then, we can easily confirm that the MMSP $\mathcal{P}$ accepts $\mathfrak{A}=$ $\{\mathcal{A} \subset[\mathrm{n}]|| \mathcal{A} \mid \geq \mathrm{r}\}$ and rejects $\mathfrak{B}=\{\mathcal{B} \subset[\mathrm{n}]|| \mathcal{B} \mid \leq \mathrm{t}\}$ as follows. For any permutation $\pi$ of $[\mathrm{n}]$, fix $\mathcal{A}:=\{\pi(i) \mid i \in$ $[\mathrm{r}]\} \in \mathfrak{A}$ and $\mathcal{B}:=\{\pi(i) \mid i \in[\mathrm{t}]\} \in \mathfrak{B}$. The matrices

$$
\begin{aligned}
& \mathrm{S}=\mathrm{G}_{\tau^{-1}(\mathcal{A})}=\mathbf{G}_{\mathcal{A}}=\left(\begin{array}{cccc}
1 & \alpha_{1}^{\pi(1)} & \cdots & \alpha_{\mathrm{r}-1}^{\pi(1)} \\
\vdots & \vdots & \ddots & \vdots \\
1 & \alpha_{1}^{\pi(\mathrm{r})} & \cdots & \alpha_{\mathrm{r}-1}^{\pi(\mathrm{r})}
\end{array}\right) \in \mathbb{F}_{q}^{r \times r}, \\
& \mathbf{T}=\left(\begin{array}{cc}
\mathrm{I}_{\mathrm{r}-\mathrm{t}} & 0 \\
\mathbf{G}_{\tau^{-1}(\mathcal{B})}
\end{array}\right)=\left(\begin{array}{cc}
\mathrm{I}_{\mathrm{r}-\mathrm{t}} & 0 \\
\mathrm{G}_{\mathcal{B}}
\end{array}\right)=\left(\begin{array}{cccc} 
& \mathrm{I}_{\mathrm{r}-\mathrm{t}} & & 0 \\
1 & \alpha_{1}^{\pi(1)} & \cdots & \alpha_{\mathrm{r}-1}^{\pi(1)} \\
\vdots & \vdots & \ddots & \vdots \\
1 & \alpha_{1}^{\pi(\mathrm{t})} & \cdots & \alpha_{\mathrm{r}-1}^{\pi(\mathrm{t})}
\end{array}\right) \in \mathbb{F}_{q}^{r \times \mathrm{r}}
\end{aligned}
$$

are invertible matrices. We obtain the acceptance of $\mathcal{P}$ because the invertibility of $S$ implies that the row span of $S$ is $\mathbb{F}_{q}^{r}$ and thus includes $\mathcal{E}=\left\{\mathbf{e}_{1}, \ldots, \mathbf{e}_{\mathrm{r}-\mathrm{t}}\right\}$. We obtain the rejection of $\mathcal{P}$ because the row vectors of $\mathrm{T}$ are linearly independent and thus, the span of the last $t$ row vectors does not include the span of $\mathcal{E}$.

Thus, the CSSPIR protocol $\hat{\Phi}_{\text {SPIR }}^{m}[\mathcal{P}]$ defined in Protocol 3 is an $(\mathrm{r}, \mathrm{t}, \mathrm{n})$-SPIR protocol with the PIR rate $R_{\text {PIR }}=(\mathrm{r}-\mathrm{t}) / \mathrm{n}$. A linear $(\mathrm{r}, \mathrm{t}, \mathrm{n})$-CSNSS protocol with the SS rate $R_{\mathrm{SS}}=\mathrm{r}-\mathrm{t}$ can also be constructed by the equivalence in Corollary 5 , Given $t, r, n$, these protocols are optimal from Proposition 1 and Corollary 4

\section{CONCLUSION}

We have studied the equivalence relation between nonperfect secret sharing and symmetric private information retrieval. We have defined the two protocols with access structures, which represent the authorized and forbidden sets for non-perfect secret sharing and the response and collusion patterns for symmetric private information retrieval. We first showed that any SPIR protocols can be converted into NSS protocols. From this relation, we proved an upper bound of CSSPIR capacity with arbitrary response and collusion patterns. Next, we proved the equivalence of linear CSNSS, linear CSSPIR, and MMSP. From this implication, we obtained the existence of CSSPIR for any access structure.

\section{APPENDIX A \\ Proof of THEOREM 1}

We separately prove $(a) \Longrightarrow(b)$ and $(b) \Longrightarrow(a)$ of Theorem 1

Step $1(a) \Longrightarrow(b)$ : It follows from the rejection condition of the MMSP that any $t$ rows of $G^{\prime \prime}$ are linearly independent. Thus, $G^{\prime \prime}$ is the generator matrix of an (n, t)-MDS code. Also, we can show that any $r$ rows of $G$ are linearly independent from the acceptance condition of the MMSP and the MDS property of G". To be precise, without losing generality, we prove that $\mathrm{G}_{[r]} \in \mathbb{F}_{q}^{r \times r}$ is invertible as follows.

The acceptance condition implies span $\mathcal{E} \subset$ rowspan $G_{[r]}$. Thus, $\operatorname{span} \mathcal{E} \oplus \operatorname{rowspan} \mathrm{G}_{[\mathrm{t}]} \subset \operatorname{rowspan} \mathrm{G}_{[\mathrm{r}]}$. On the other hand, the rejection condition implies rowspan $G_{[t]} \cap$ rowspan $\mathcal{E}=\{0\}$. Thus, we have $r=\operatorname{dim}(\operatorname{span} \mathcal{E} \oplus$ rowspan $\left.\mathrm{G}_{[\mathrm{t}]}\right) \leq \operatorname{dim} \mathrm{G}_{[\mathrm{r}]} \leq \mathrm{r}$, which implies $\mathrm{G}_{[\mathrm{r}]}$ is invertible. Step $2(b) \Longrightarrow(a)$ : We prove in the following that $G$ and $G^{\prime \prime}$ with condition (b) satisfy the acceptance and rejection conditions of the MMSP $\mathcal{P}$. Since any $r$ rows of $G \in \mathbb{F}_{q}^{\text {nxr }}$ are linearly independent, the space spanned by those rows is $\mathbb{F}_{q}^{r}$, which implies the acceptance condition of the MMSP $\mathcal{P}$.

Next, we prove the rejection condition of $\mathcal{P}$ from the MDS property of $G^{\prime \prime} \in \mathbb{F}_{q}^{\text {nxt }}$ by contradiction. Suppose that the rejection condition does not hold, i.e., there exists a row vector $\mathbf{x} \in \mathbb{F}_{q}^{\mathrm{n}}$ and a set $\mathcal{B}$ with $|\mathcal{B}|=\mathrm{t}$ satisfying $0 \neq \mathbf{x} \in \operatorname{span} \mathcal{E} \cap$ rowspan $G_{\mathcal{B}}$. Since $\mathbf{x} \in \operatorname{span} \mathcal{E}$, the last $\mathrm{t}$ coordinates of $\mathbf{x}$ are 0 . On the other hand, since $G_{\mathcal{B}}^{\prime \prime} \in \mathbb{F}_{q}^{\mathrm{t} \times \mathrm{t}}$ is invertible, the last $t$ coordinates of $\mathbf{x}$ are 0 if and only if $\mathbf{x}=0$, which is a contradiction. Thus, the rejection condition holds. 


\section{APPENDIX B}

PROOF OF LEMMA 2

In this section, we prove of Lemma 2. In the following subsections, we separately prove the following inequalities.

$$
\begin{aligned}
& I\left(M_{[2: \mathrm{f}]} ; M_{1} D \mid R=r^{*}, K=1\right) \\
& \leq \beta+h_{2}(1-\alpha)+(1-\alpha) \log \mathrm{m}+\log \alpha, \\
& I\left(M_{1} ; D_{\mathcal{B}} \mid R=r^{*}, K=1\right) \\
& \leq \beta+4 \sqrt{2 \gamma \mathrm{f}} \log \mathrm{m}+2 h_{2}(\sqrt{2 \gamma \mathrm{f}}) .
\end{aligned}
$$

Then, with these two inequalities, we obtain the lemma as

$$
\begin{aligned}
& I\left(M_{1} ; D_{\mathcal{B}} \mid M_{[2: f]}, R=r^{*}, K=1\right) \\
& \leq I\left(M ; D_{\mathcal{B}} \mid R=r^{*}, K=1\right) \\
& =I\left(M_{1} ; D_{\mathcal{B}} \mid R=r^{*}, K=1\right)+I\left(M_{[2: f]} ; D \mid M_{1}, R=r^{*}, K=1\right) \\
& =I\left(M_{1} ; D_{\mathcal{B}} \mid R=r^{*}, K=1\right)+I\left(M_{[2: f]} ; M_{1} D \mid R=r^{*}, K=1\right) \\
& \leq \xi(\alpha, \beta, \gamma),
\end{aligned}
$$

where the last inequality follows from (71) and (72).

\section{A. Proof of (71)}

For the proof of 71 , we prove the following lemma.

\section{Lemma 5. Suppose}

- $A, B$ are independent,

- $B$ is recovered from $C$ with probability $\alpha$, and

- $I(A ; C) \leq \beta$.

Then, we have

$$
I(A ; B C) \leq \beta+h_{2}(1-\alpha)+(1-\alpha) \log |\mathcal{B}|+\log \alpha,
$$

where $\mathcal{B}$ is the space of $B$.

By applying the above lemma for the case of $(A, B, C)=$ ( $\left.M_{[2: \mathrm{ff}}, M_{1}, D\right)$ while conditioning $R=r^{*}, K=1$, Eq. (71) is obtained as

$$
\begin{aligned}
& I\left(M_{[2: f]} ; M_{1} D_{\mathcal{B}} \mid R=r^{*}, K=1\right) \\
& \leq I\left(M_{[2: f]} ; M_{1} D \mid R=r^{*}, K=1\right) \\
& \leq \beta+h_{2}(1-\alpha)+(1-\alpha) \log \mathrm{m}+\log \alpha .
\end{aligned}
$$

Proof of Lemma 5 From Fano's inequality, we have

$$
H(B \mid C) \leq \beta+h_{2}(1-\alpha)+(1-\alpha) \log |\mathcal{B}| .
$$

On the other hand, from the lower bound of the guessing probability [57], [58], we have

$$
H(B \mid A C) \geq-\log \alpha .
$$

Thus, combining the above two inequalities, we obtain the desired inequality as

$$
\begin{aligned}
I(A ; B C) & =I(A ; C)+I(A ; B \mid C) \\
& =I(A ; C)+H(B \mid C)-H(B \mid A C) \\
& \leq 2 \beta+h_{2}(1-\alpha)+(1-\alpha) \log |\mathcal{B}|+\log \alpha .
\end{aligned}
$$

\section{B. Proof of (72)}

For the proof, we define the variational distance

$$
d(p, q):=\sum_{x}|p(x)-q(x)| .
$$

Throughout this section, we denote the distribution of a random variable $X$ by $P_{X}$ and the probability $P_{X}(x)$ by $P_{X}$, i.e., subscript with the lowercase letter of $X$.

First, we prepare the following proposition.

Proposition 4 (Classical Alicki-Fannes inequality [60]). Let $X, Y$ be random variable on $X, Y$ and $p, q$ be probability distributions on $\mathcal{X} \times \mathcal{Y}$ such that $\epsilon:=d(p, q)=\sum_{x, y} \mid p(x, y)-$ $q(x, y) \mid$. Then,

$$
\left|H\left(p_{X \mid Y}\right)-H\left(q_{X \mid Y}\right)\right| \leq 4 \epsilon \log |X|+2 h_{2}(\epsilon) .
$$

Remark 6. In [60], Proposition 44 is originally proved for quantum systems and states. By restricting the quantum systems and states as classical systems and random variables, we directly obtain Proposition 4

Now, we prove (72).

Proof of (72). We prove the lemma by two steps.

Step 1: First, we prove

$$
d\left(P_{M_{1} D_{\mathcal{B}} Q_{\mathcal{B}} \mid K=1}, P_{M_{1} D_{\mathcal{B}} Q_{\mathcal{B}} \mid K=2}\right) \leq \sqrt{2 \gamma f}
$$

for any $\mathcal{B} \in \mathfrak{B}$. This inequality for the threshold case is proved in [61, Lemma 5]. With the similar idea, we give the proof of (89). We have

$$
\begin{aligned}
\gamma & \geq I\left(K ; Q_{\mathcal{B}}\right) \\
& =D\left(P_{K Q_{\mathcal{B}}} \| P_{K} \times P_{Q_{\mathcal{B}}}\right) \\
& \stackrel{(a)}{=} \frac{1}{\mathrm{f}} \sum_{k} D\left(P_{Q_{\mathcal{B}} \mid K=k} \| P_{Q_{\mathcal{B}}}\right) \\
& \stackrel{(b)}{\geq} \frac{2}{\mathrm{f}} \sum_{k} d^{2}\left(P_{Q_{\mathcal{B}} \mid K=k}, P_{Q_{\mathcal{B}}}\right) \\
& \geq \frac{2}{\mathrm{f}} d^{2}\left(P_{Q_{\mathcal{B}} \mid K=k^{\prime}}, P_{Q_{\mathcal{B}}}\right) \\
& \stackrel{(c)}{=} \frac{2}{\mathrm{f}} d^{2}\left(P_{M_{1} D_{\mathcal{B}} Q_{\mathcal{B}} \mid K=k^{\prime}}, P_{M_{1} D_{\mathcal{B}} Q_{\mathcal{B}}}\right)
\end{aligned}
$$

for any $k^{\prime} \in[\mathrm{f}]$. The equality $(a)$ follows from the uniform randomness of $K$, and the inequality $(b)$ follows from Pinsker's inequality. The equality $(c)$ follows from

$$
\begin{aligned}
& d\left(P_{M_{1} D_{\mathcal{B}} Q_{\mathcal{B}} \mid K=k}, P_{M_{1} D_{\mathcal{B}} Q_{\mathcal{B}}}\right) \\
& =\frac{1}{2} \sum_{m_{1}, a_{\mathcal{B}}, q_{\mathcal{B}}, k}\left|P_{m_{1} a_{\mathcal{B}} q_{\mathcal{B}} \mid k}-P_{m_{1} a_{\mathcal{B}} q_{\mathcal{B}}}\right| \\
& =\frac{1}{2} \sum_{m_{1}, a_{\mathcal{B}}, q_{\mathcal{B}}, k}\left|P_{m_{1} a_{\mathcal{B}} \mid k q_{\mathcal{B}}} P_{q_{\mathcal{B}} \mid k}-P_{m_{1} a_{\mathcal{B}} \mid q_{\mathcal{B}}} P_{q_{\mathcal{B}}}\right| \\
& \stackrel{(\text { d })}{=} \frac{1}{2} \sum_{m_{1}, a_{\mathcal{B}}, q_{\mathcal{B}}, k}\left|P_{m_{1} a_{\mathcal{B}} \mid q_{\mathcal{B}}} P_{q_{\mathcal{B}} \mid k}-P_{m_{1} a_{\mathcal{B}} \mid q_{\mathcal{B}}} P_{q_{\mathcal{B}}}\right| \\
& =\frac{1}{2} \sum_{q_{\mathcal{B}}, k}\left|P_{q_{\mathcal{B}} \mid k}-P_{q_{\mathcal{B}}}\right| \\
& =d\left(P_{\mathcal{Q}_{\mathcal{B}} \mid K=k}, P_{Q_{\mathcal{B}}}\right)
\end{aligned}
$$


where the inequality $(d)$ holds since $K-Q_{\mathcal{B}}-M_{1} D_{\mathcal{B}}$ is a Markov chain. Then, from Eq. (95) and the triangular inequality, we obtain (89) as

$$
\begin{aligned}
\sqrt{2 \gamma f} \geq & d\left(P_{M_{1} D_{\mathcal{B}} Q_{\mathcal{B}} \mid K=1}, P_{M_{1} D_{\mathcal{B}} Q_{\mathcal{B}}}\right) \\
& +d\left(P_{M_{1} D_{\mathcal{B}} Q_{\mathcal{B}} \mid K=2}, P_{M_{1} D_{\mathcal{B}} Q_{\mathcal{B}}}\right) \\
\geq & d\left(P_{M_{1} D_{\mathcal{B}} Q_{\mathcal{B}} \mid K=1}, P_{M_{1} D_{\mathcal{B}} Q_{\mathcal{B}} \mid K=2}\right) .
\end{aligned}
$$

Step 2: Next, we prove the desired inequality

$$
I\left(M_{1} ; D_{\mathcal{B}} \mid R=r^{*}, K=1\right) \leq \beta+g(\gamma, \mathrm{m}),
$$

where

$$
g(\gamma, \mathrm{m}):=4 \sqrt{2 \gamma \mathrm{f}} \mathrm{m}+2 h_{2}(\sqrt{2 \gamma \mathrm{f}}) .
$$

We have

$$
\begin{aligned}
& \left|I\left(M_{1} ; D_{\mathcal{B}} \mid R, K=1\right)-I\left(M_{1} ; D_{\mathcal{B}} \mid R, K=2\right)\right| \\
& \stackrel{(a)}{=}\left|I\left(M_{1} ; D_{\mathcal{B}} \mid Q_{\mathcal{B}}, K=1\right)-I\left(M_{1} ; D_{\mathcal{B}} \mid Q_{\mathcal{B}}, K=2\right)\right| \\
& =\left|H\left(M_{1} \mid D_{\mathcal{B}} Q_{\mathcal{B}}, K=1\right)-H\left(M_{1} \mid D_{\mathcal{B}} Q_{\mathcal{B}}, K=2\right)\right| \\
& \stackrel{(b)}{\leq} 4 \sqrt{2 \gamma f} \log |\mathcal{M}|+2 h_{2}(\sqrt{2 \gamma f}) \\
& =g(\gamma, \mathrm{m}),
\end{aligned}
$$

where $(a)$ holds because $R-Q_{\mathcal{B}}-M_{i} D_{\mathcal{B}}$ is a Markov chain and $(b)$ is obtained by combining Proposition 4 and (89). Rearranging the inequality (111), we have

$$
\begin{aligned}
I\left(M_{1} ; D_{\mathcal{B}} \mid R, K=1\right) & \leq I\left(M_{1} ; D_{\mathcal{B}} \mid R, K=2\right)+g(\gamma, \mathrm{m}) \\
& \leq I\left(M_{[f] \backslash 2} ; D \mid R, K=2\right)+g(\gamma, \mathrm{m}) \\
& \stackrel{(c)}{\leq} \beta+g(\gamma, \mathrm{m}),
\end{aligned}
$$

where $(c)$ follows from the server secrecy of $\Phi_{\mathrm{SPIR}}^{\mathrm{m}}$. Since $r^{*}$ is defined in (17) to satisfy the inequality

$$
\begin{aligned}
& I\left(M_{1} ; D_{\mathcal{B}} \mid R=r^{*}, K=1\right) \\
& \leq \sum_{r \in \mathcal{R}} P_{R}(r) I\left(M_{1} ; D_{\mathcal{B}} \mid R=r, K=1\right) \\
& =I\left(M_{1} ; D_{\mathcal{B}} \mid R, K=1\right),
\end{aligned}
$$

the inequality (112) derives the desired inequality

$$
I\left(M_{1} ; D_{\mathcal{B}} \mid R=r^{*}, K=1\right) \leq \beta+g(\gamma, \mathrm{m}) .
$$

\section{REFERENCES}

[1] S. Song and M. Hayashi, "Equivalence of Non-Perfect Secret Sharing and Symmetric Private Information Retrieval with General Access Structure," Proceedings of 2021 IEEE International Symposium on Information Theory, in press.

[2] A. Shamir, "How to share a secret," Communications of the ACM, 22(11):612-613, 1979.

[3] G.R. Blakley, "Safeguarding Cryptographic Keys," Managing Requirements Knowledge, International Workshop on (AFIPS), 48: 313-317, 1979.

[4] B. Chor, O. Goldreich, E. Kushilevitz, and M. Sudan, "Private information retrieval," Journal of the ACM, 45(6):965-981, 1998.

[5] Y. Gertner, Y. Ishai, E. Kushilevitz, and T. Malkin. "Protecting data privacy in private information retrieval schemes," Journal of Computer and Systems Sciences, 60(3):592-629, 2000. Earlier version in STOC 98.
[6] A. Beimel, Y. Ishai, E. Kushilevitz, and I. Orlov, "Share Conversion and Private Information Retrieval," 2012 IEEE 27th Conference on Computational Complexity (CCC), 2012.

[7] L. Li, M. Militzer, and A. Datta, "rPIR: ramp secret sharing-based communication-efficient private information retrieval," Int. J. Inf. Secur. 16, 603-625, 2017.

[8] H. Yang, W. Shin, and J. Lee, "Private Information Retrieval for Secure Distributed Storage Systems," IEEE Transactions on Information Forensics and Security, pp.(99):1-1, 2018.

[9] R. G.L. D'Oliveira and S. El Rouayheb, "Lifting Private Information Retrieval from Two to any Number of Messages," Proc. IEEE Int. Symp. Inf. Theory (ISIT), pp. 1744-1748, 2018.

[10] G.R. Blakley and C.A. Meadows, "Security of ramp schemes," CRYPTO 1984, Lecture Notes in Computer Science 196, Springer, pp.242-268, 1985.

[11] H. Yamamoto, "On secret sharing systems using $(k, L, n)$ threshold scheme," IECE. Trans., J68-A(9):945-952, 1985. (in Japanese). English translation: Electronics and Communications in Japan, Part I, vol. 69, no. 9, pp. 46-54, Scripta Technica, Inc., 1986.

[12] W. Ogata, K. Kurosawa, and S. Tsujii, "Nonperfect Secret Sharing Schemes," Advances in Cryptology, Auscrypt 92, Lecture Notes in Comput. Sci., 718, 56-66, 1993.

[13] K. Okada and K. Kurosawa, "Lower Bound on the Size of Shares of Nonperfect Secret Sharing Schemes," Advances in Cryptology, Asiacrypt 94, Lecture Notes in Comput. Sci., 917, 33-41, 1995.

[14] P. Paillier, "On ideal non-perfect secret sharing schemes," Security Protocols, 5th International Workshop, Lecture Notes in Comput. Sci., 1361, 207-216, 1998.

[15] M. Yoshida, T. Fujiwara and M. P. C. Fossorier, "Optimal Uniform Secret Sharing," IEEE Transactions on Information Theory, vol. 65, no. 1, pp. 436-443, 2019.

[16] O. Farràs, T. Hansen, T. Kaced, C. Padró, "On the information ratio of non-perfect secret sharing schemes," Algorithmica, , vol. 79, no. 4, pp. 987-1013, 2017.

[17] H. Sun and S. Jafar, "The Capacity of Symmetric Private Information Retrieval," 2016 IEEE Globecom Workshops (GC Wkshps), Washington, DC, 2016, pp. 1-5.

[18] H. Sun and S. Jafar, "The capacity of private information retrieval," IEEE Transactions on Information Theory, vol. 63, no. 7, pp. 40754088, 2017.

[19] Z. Jia, H. Sun, and S. A. Jafar, "The capacity of private information retrieval with disjoint colluding sets," Proceedings of 2017 IEEE Global Communications Conference, 2017.

[20] R. Tajeddine, O. W Gnilke, D. Karpuk, R. Freij-Hollanti, C. Hollanti, and S. El Rouayheb, "Private information retrieval schemes for coded data with arbitrary collusion patterns," 2017 IEEE International Symposium on Information Theory (ISIT), pp. 1908-1912, 2017.

[21] Y. Zhang and G. Ge, "Private information retrieval from MDS coded databases with colluding servers under several variant models," arXiv preprint: 1705.03186, 2017.

[22] H. Sun and S. A. Jafar, "Private information retrieval from MDS coded data with colluding servers: Settling a conjecture by Freij-Hollanti et al.," IEEE Transactions on Information Theory, 64(2):1000-1022, 2018.

[23] X. Yao, N. Liu, and W. Kang, "The Capacity of Private Information Retrieval Under Arbitrary Collusion Patterns," Proceedings of 2020 IEEE International Symposium on Information Theory (ISIT), pp. 10411046,2020

[24] J. Cheng, N. Liu, and W. Kang, "The Capacity of Symmetric Private Information Retrieval under Arbitrary Collusion and Eavesdropping Patterns," arXiv:2010.08249 2020.

[25] K. Banawan and S. Ulukus, "The Capacity of Private Information Retrieval from Byzantine and Colluding Databases," IEEE Transactions on Information Theory, vol. 65, no. 2, pp. 1206-1219, 2019.

[26] Q. Wang and M. Skoglund, "Secure private information retrieval from colluding databases with eavesdroppers," Proc. IEEE Int. Symp. Inf. Theory (ISIT), pp. 2456-2460, 2018.

[27] L. Holzbaur, R. Freij-Hollanti, J. Li, and C. Hollanti, "Towards the capacity of private information retrieval from coded and colluding servers," arXiv:1903.12552v6, 2021.

[28] R. Tajeddine, O. W. Gnilke, D. Karpuk, R. Freij-Hollanti and C. Hollanti, "Private Information Retrieval From Coded Storage Systems With Colluding, Byzantine, and Unresponsive Servers," IEEE Transactions on Information Theory, vol. 65, no. 6, pp. 3898-3906, 2019.

[29] E. F. Brickell, "Some ideal secret sharing schemes," Journal of Combin. Math. and Combin. Comput., 6:105-113, 1989.

[30] M. Karchmer and A. Wigderson, "On span programs," Proc. of the 8th IEEE Structure in Complexity Theory, pp. 102-111, 1993. 
[31] A. Beimel, "Secure Schemes for Secret Sharing and Key Distribution," PhD thesis, Technion, 1996. www.cs.bgu.ac.il/ beimel/pub.html.

[32] A. Beimel, "Secret-Sharing Schemes: A Survey," Chee Y.M. et al. (eds) Coding and Cryptology. IWCC 2011. Lecture Notes in Computer Science, vol 6639, Springer, Berlin, Heidelberg, 2011.

[33] M. Bertilsson and I. Ingemarsson. A construction of practical secret sharing schemes using linear block codes. In J. Seberry and Y. Zheng, editors, Advances in Cryptology - AUSCRYPT '92, volume 718 of Lecture Notes in Computer Science, pp. 67-79. Springer-Verlag, 1993.

[34] M. van Dijk. A linear construction of perfect secret sharing schemes. In A. De Santis, editor, Advances in Cryptology - EUROCRYPT '94, volume 950 of Lecture Notes in Computer Science, pp. 23-34. SpringerVerlag, 1995.

[35] G. Blakley and G. Kabatianski, "Ideal perfect threshold schemes and MDS codes," IEEE International Symposium on Information Theory, vol. 4, pp. 77843, 1995.

[36] H. Chen, R. Cramer, S. Goldwasser, R. de Haan, and V. Vaikuntanathan, "Secure computation from random error correcting codes," Advances in Cryptology (Lecture Notes in Computer Science), vol. 4515. Heidelberg, Germany: Springer-Verlag, pp. 291-310, 2007.

[37] I. M. Duursma and S. Park, "Coset bounds for algebraic geometric codes," Finite Fields Appl., vol. 16, no. 1, pp. 36-55, Jan. 2010.

[38] J. Kurihara, T. Uyematsu, and R. Matsumoto, "Secret sharing schemes based on linear codes can be precisely characterized by the relative generalized Hamming weight," IEICE Trans. Fundam. Electron., Commun., Comput. Sci., vol. E95-A, no. 11, pp. 2067-2075, Nov. 2012.

[39] W. Huang and J. Bruck, "Secret sharing with optimal decoding and repair bandwidth," 2017 IEEE International Symposium on Information Theory (ISIT), pp. 1813-1817, 2017.

[40] Q. Wang and M. Skoglund, "Symmetric private information retrieval for MDS coded distributed storage," Proceedings of 2017 IEEE International Conference on Communications (ICC), pp. 1-6, 2017.

[41] Q. Wang and M. Skoglund, "Secure Symmetric Private Information Retrieval from Colluding Databases with Adversaries," 2017 55th Annual Allerton Conference on Communication, Control, and Computing (Allerton), pp. 1083-1090, 2017.

[42] K. Banawan and S. Ulukus, "The Capacity of Private Information Retrieval from Coded Databases," IEEE Transactions on Information Theory, vol. 64, no. 3, 2018.

[43] L. H. Ozarow and A. D. Wyner, "Wire-tap-channel II," AT\&T Bell Labs Tech. J., 63:2135-2157, 1984.

[44] A. Subramanian, Steven W. McLaughlin, "MDS codes on the erasureerasure wiretap channel" arXiv preprint, arXiv:0902.3286 2009.

[45] N. Cai and R. W. Yeung, "Secure Network Coding on a Wiretap Network," IEEE Trans. Inform. Theory, vol. 57, no. 1, 424-435, 2011.

[46] N. Cai and M. Hayashi, "Secure Network Code for Adaptive and Active Attacks with No-Randomness in Intermediate Nodes," IEEE Transactions on Information Theory, vol. 66, 1428-1448, 2020

[47] S. El Rouayheb, E. Soljanin, and A. Sprintson, "Secure network coding for wiretap networks of type II," IEEE Trans. Inf. Theory, vol. 58, no. 3, pp. 1361-1371, Mar. 2012

[48] C.-K. Ngai, R. W. Yeung, and Z. Zhang, "Network generalized Hamming weight," IEEE Trans. Inf. Theory, vol. 57, no. 2, pp. 1136-1143, Feb. 2011.

[49] D. Silva and F. R. Kschischang, "Universal secure network coding via rank-metric codes," IEEE Trans. Inf. Theory, vol. 57, no. 2, pp. 1124 1135, Feb. 2011.

[50] Z. Zhang and B. Zhuang, "An application of the relative network generalized Hamming weight to erroneous wiretap networks," Proc. IEEE Inf. Theory Workshop, Taormina, Italy, pp. 70-74, Oct. 2009.

[51] J. Kurihara, R. Matsumoto and T. Uyematsu, "Relative Generalized Rank Weight of Linear Codes and Its Applications to Network Coding," IEEE Transactions on Information Theory, vol. 61, no. 7, pp. 3912-3936, July 2015.

[52] A. G. Dimakis, P. B. Godfrey, Y. Wu, M. J. Wainwright and K. Ramchandran, "Network Coding for Distributed Storage Systems," IEEE Transactions on Information Theory, vol. 56, no. 9, pp. 4539-4551, 2010.

[53] S. Vaudenay, "On the Need for Multipermutations: Cryptanalysis of MD4 and SAFER" 2nd International Workshop on Fast Software Encryption (FSE '94), Leuven: Springer-Verlag, pp. 286-297, 1994.

[54] R. Zamir, S. Shamai, and U. Erez, "Nested linear/lattice codes for structured multiterminal binning," IEEE Trans. Inf. Theory, vol. 48, no. 6, pp. 1250-1276, Jun. 2002.

[55] T. H. Chan, S.-W. Ho, and H. Yamamoto, "Private information retrieval for coded storage," in Proceedings of 2015 IEEE International Symposium on Information Theory (ISIT), pp. 2842-2846, June 2015.
[56] R. Freij-Hollanti, O. W. Gnilke, C. Hollanti, and D. A. Karpuk, "Private information retrieval from coded databases with colluding servers," SIAM J. Appl. Algebra Geometry, vol. 1, no. 1, pp. 647-664, 2017.

[57] H. Buhrman, M. Christandl, P. Hayden, H.-K. Lo, and S. Wehner, "Security of quantum bit string commitment de- pends on the information measure," Phys. Rev. Lett., vol. 97, no. 25, p. 250501, 2006.

[58] R. Konig, R. Renner and C. Schaffner, "The Operational Meaning of Min- and Max-Entropy," IEEE Transactions on Information Theory, vol. 55, no. 9, pp. 4337-4347, 2009.

[59] I. S. Reed, G. Solomon, "Polynomial Codes over Certain Finite Fields," Journal of the Society for Industrial and Applied Mathematics, 8 (2): 300-304, 1960.

[60] R. Alicki and M. Fannes, "Continuity of quantum mutual information," J. of Phys. A: Math. and Gen., 37(5):L55-L57, 2004.

[61] S. Song and M. Hayashi, "Capacity of Quantum Private Information Retrieval with Colluding Servers," IEEE Transactions on Information Theory, vol. 67, no. 8, pp. 5491-5508, 2021. 